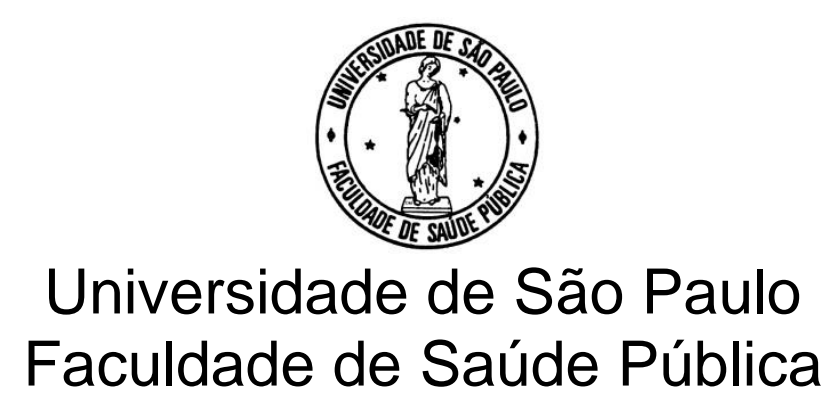

Distribuição espacial e temporal dos casos de tuberculose pulmonar em municípios do Estado de São Paulo, 2007 a 2013

Erico Filev Maia

Dissertação apresentada à Faculdade de Saúde Pública da Universidade de São Paulo, para obtenção do título de Mestre em Ciências, Programa de Pós Graduação em Saúde Pública

Área de concentração: Epidemiologia

Orientador: Prof. Dr. Francisco

Chiaravalloti Neto

São Paulo

2017 


\section{Distribuição espacial e temporal dos casos de tuberculose pulmonar em municípios do Estado de São Paulo, 2007 a 2013}

Erico Filev Maia

Dissertação apresentada à Faculdade de Saúde Pública da Universidade de São Paulo, para obtenção do título de Mestre em Ciências, Programa de Pós Graduação em Saúde Pública

Área de concentração: Epidemiologia

Orientador: Prof. Dr. Francisco

Chiaravalloti Neto 
Autorizo a reprodução e divulgação total ou parcial deste trabalho, por qualquer meio convencional ou eletrônico, para fins de estudo e pesquisa, desde que citada a fonte. 
Aos meus pais, Marco Antonio e Maria Aparecida, cujos exemplos me ensinaram que a perseverança é um caminho a se trilhar...

À minha esposa, Laura, cuja parceria, carinho e apoio foram fundamentais nessa construção... 


\section{AGRADECIMENTOS}

Ao meu orientador, Prof. Dr. Francisco Chiaravalloti Neto, pela acolhida desde o primeiro contato, pela oportunidade de ensinamento e pesquisa, por me incentivar a adquirir novas habilidades profissionais, pela generosidade, e, principalmente, por continuar acreditando em mim diante todos os desafios que surgiram nesse período.

À Prof ${ }^{a}$. Dr ${ }^{a}$. Inês Fronteira, pela forma generosa com que compartilhou seu conhecimento e engrandeceu esse trabalho.

À Prof ${ }^{a}$. Dr ${ }^{a}$. Ana Paula Sayuri Sato, pelos ensinamentos que ultrapassaram a sala de aula e contribuíram para o crescimento desse trabalho.

Ao Prof. Dr. Ricardo Alexandre Arcêncio, pela valiosa contribuição desde a qualificação desse trabalho. Seus ensinamentos e suas obras foram fundamentais para essa construção.

À Prof ${ }^{a}$. Dr ${ }^{a}$. Alessandra Cristina Guedes Pellini, cujas observações e o conhecimento da construção de um trabalho científico foram de especial importância para essa dissertação.

Ao Prof. Dr. Oswaldo Santos Baquero, que em diversas oportunidades contribuiu para que não se perdesse o norte dessa pesquisa. 


\section{RESUMO}

Introdução: A tuberculose (TB) ainda é uma doença em posição de destaque na saúde pública, mantendo-se como emergência global desde 1993, devido ao elevado número de infectados pelo bacilo causador, e devido a se apresentar como uma das principais causas de morte no planeta. Objetivos: Descrever a distribuição espacial e temporal dos casos de TB pulmonar (TBp) no estado de São Paulo (ESP) entre 2007 e 2013 e e avaliar a relação desta com indicador de condições socioeconômicas e demográficas. Método: Tratase de estudo ecológico, cujas informações foram obtidas nos bancos de dados do Sistema de Informação de Agravos de Notificação, do Departamento de Informática do Sistema Único de Saúde e do Instituto Brasileiro de Geografia e Estatística (IBGE). Os casos de TBp foram georreferenciados a partir dos códigos dos municípios de residência dos doentes. Calcularam-se incidências, no tempo e no espaço, e produziram-se mapas temáticos, identificando áreas de maior e menor risco da ocorrência da doença. A incidência de TBp foi relacionada a indicador socioeconômico e demográfico por meio de regressão espacial. As análises estatísticas utilizaram método de Prais-Winsten, estatísticas de varredura e modelagem Bayesiana Gaussiana latente. Resultados: Foram notificados 77.390 casos de TBp em pacientes, desconsiderando casos em presidiários ou coinfecção com vírus da imunodeficiência humana. A taxa de incidência para todo o período de estudo foi igual a 26,8 casos/100.000 habitantes-ano. As taxas de incidência total, masculina e feminina apresentaram estacionariedade entre 2007 e 2013. Os homens e pessoas entre 15 e 59 anos apresentaram excesso de risco. Os municípios das mesorregiões Metropolitana de São Paulo e Litoral Sul Paulista apresentaram, em geral e em todos os anos de estudo, altas probabilidades de que suas razões de incidência padronizada (RIP) fossem maiores do que a unidade e parte deles constitui-se como um aglomerado de alto risco para ocorrência de TBp. Municípios das mesorregiões de Presidente Prudente, Bauru e Marília apresentaram tendência temporal crescente das incidências em relação ao restante dos municípios do ESP. Municípios com baixas renda, escolaridade e longevidade apresentaram risco para ocorrência de TBp 25,0\% superior aos municípios com alta renda e altas e médias escolaridade e longevidade. Conclusão: As taxas de incidência apresentaram, entre 2007 e 2013, estacionariedade e maiores valores para homens e pessoas entre 15 e 59 anos. Identificaram-se municípios com alto risco para ocorrência de TBp e municípios, embora com riscos médios para TBp, com tendência crescente das incidências. Municípios com piores condições socioeconômicas e demográficas relevaram-se de maior risco para ocorrência do agravo.

Palavras chave: Tuberculose, Incidência, Análise Espacial, Distribuição Temporal, Brasil. 


\section{ABSTRACT}

Introduction: Tuberculosis (TB) is still a prominent disease in public health, having remained as international emergencies since 1993, due to the high number of infected by the causative bacillus, and due to a presentation as a leading cause of death in planet. Objectives: To describe a spatial and temporal distribution of cases of pulmonary TB (TBp) in the São Paulo state between 2007 and 2013 and to evaluate its relationship with and indicator of socioeconomic and demographic conditions. Method: This is an ecological study, whose information is obtained in the databases of the Information System of Notifiable Diseases, Department of Informatics of the Unified Health System of the Brazilian Institute of Geography and Statistics (IBGE). The cases of TBp were georeferenced from the codes of the municipalities of residence of the patients. Incidence, time and space were calculated, and thematic maps were produced, identifying areas of greater and lesser risk of the occurrence of the disease. The incidence of TBp was related to socioeconomic and demographic indicators through spatial regression. Statistical analyzes used the Prais-Winsten method, scanning statistics and latent Gaussian Bayesian model. Results: A total of 77.390 cases of TBp were reported in patients, disregarding cases in prison inmates or co infection with acquired immunodeficiency syndrome. The incidence rate for the entire study period was 26.8 cases / 100,000 population-years. As total incidence rates, male and female presented stationarity between 2007 and 2013. Men and people between 15 and 59 years old presented an excess risk. The municipalities of the Metropolitan São Paulo and Litoral Sul Paulista mesoregions presented, in general and in all the years of study, high probabilities that their standardized incidence ratios (RIP) were larger than one unit and part of them constitute one Agglomerate of high risk for occurrence of TBp. Municipalities in the mesoregions of Presidente Prudente, Bauru and Marilia showed an increasing temporal trend of incidence in relation to the rest of ESP municipalities. Municipalities with low income, schooling and longevity presented a risk of occurrence of TBp $25.0 \%$ higher than municipalities with high income and high and medium schooling and longevity. Conclusion: The incidence rates between 2007 and 2013 showed stationarity and higher values for men and people between 15 and 59 years. Municipalities with high risk for occurrence of TBp and municipalities were identified, although with average risks for TBp, with an increasing tendency of incidence. Municipalities with worse socioeconomic and demographic conditions were found to be at greater risk for the occurrence of the disease.

Keywords: Tuberculosis, Incidence, Spatial Analysis, Temporal Distribution, Brazil. 


\section{ÍNDICE}

Lista de Figura $\quad 9$

$\begin{array}{ll}\text { Lista de Siglas } & 10\end{array}$

1 - Introdução 11

2 - Objetivos

2.1 - Objetivo Geral 23

2.2 - Objetivos específicos 23

3 - Métodos

3.1 - Tipo,população e área de estudo 24

3.2 - Fonte de dados 24

3.3 - Georreferenciamento 26

3.4 - Análise dos dados 26

3.5 - Aspectos éticos 30

4 - Resultados e Discussão 31

5 - Conclusões 56

6 - Referências 58

7 - Anexos

7.1 - Anexo A - Aprovação do Comitê de Ética em Pesquisa 61

7.2 - Anexo B - Confirmação da submissão do artigo à Revista de Saúde

$\begin{array}{ll}\text { Pública } & 63\end{array}$

7.3 - Anexo C - Currículo Lattes de aluno e orientador 64 


\section{LISTA DE FIGURA}

Figura 1 - América do Sul, Brasil e estado de São Paulo (A); estado de São Paulo e seus municípios, com destaque para a capital paulista (B); mesorregiões do estado de São Paulo (C). 


\section{LISTA DE SIGLAS}

BAAR - Bacilo Álcool-Ácido Resistente

DATASUS - Departamento de Informática do Sistema Único de Saúde

ESP - Estado de São Paulo

HIV - Vírus da Imunodeficiência Humana

IBGE - Instituto Brasileiro de Geografia e Estatística

iCAR - Estrutura Autorregressiva Condicional

INLA - Integrated Nested Laplace Approximation

IPRS - Índice Paulista de Responsabilidade Social

MCMC - método Markov Chain Monte Carlo

OMS - Organização Mundial da Saúde

RIP - Razão de Incidência Padronizada

SEADE - Sistema Estadual de Análise de Dados

SIDA - Síndrome da Imunodeficiência Adquirida

SIG - Sistema de Informação Geográfica

SINAN - Sistema de Informação de Agravos de Notificação

TB - Tuberculose

TBp - Tuberculose pulmonar

USP - Universidade de São Paulo 


\section{INTRODUÇÃO}

A TB é uma das doenças infecciosas conhecidas mais antigas da história da humanidade. Ainda assim, continua em posição de destaque no âmbito da saúde pública devido a ser um agravo de grande magnitude, vulnerabilidade e transcendência (MS, 2011).

A TB é uma doença crônica, que caracteristicamente cursa com granuloma e necrose. Causada pelo Mycobacterium tuberculosis, um bacilo álcool-ácido resistente (BAAR), ou pelas outras espécies Mycobacterium africanum e Mycobacterium bovis, tem os pulmões como principais sítios de infecção. (ELLNER, 2014).

A transmissão da TB ocorre através de gotículas aerossolizadas, que contenham o bacilo responsável pela doença, produzidas por indivíduos infectados, em especial aqueles indivíduos que já apresentam cavitação pulmonar, e que sejam capazes de produzir gotículas de 0,5 a $3 \mu \mathrm{m}$ de diâmetro contendo o bacilo. Essas gotículas podem permanecer suspensas por horas em ar ambiente e, se inaladas por outrem, são pequenas o suficiente para atravessar as vias aéreas e capazes de chegar aos alvéolos, porção mais distal da via respiratória. A transmissão descrita é a forma mais comum de contágio da TB, embora haja a descrição, na literatura, de inalação de aerossol gerado no ato de cuidado com feridas por abscessos de pele e tecidos moles causadas pelo $M$. tubercolusis e que são altamente infectantes (ISEMAN, 2005).

As formas extrapulmonares da TB são mais comuns em indivíduos com algum grau de imunocomprometimento, principalmente aqueles que 
apresentam coinfeccção com HIV, e crianças. Além disso, essas formas apresentam sintomas diferentes, conforme os órgãos acometidos. Cabe ressaltar que a TB pleural é a forma mais comum, entre as formas extrapulmonares, em adultos jovens HIV soronegativos. A forma ganglionar periférica é a mais frequente em crianças e indivíduos HIV soropositivos. A forma meningoencefálica responde por 3\% dos casos de TB em indivíduos HIV soronegativos, e esse número aumenta para $10 \%$ quando observado em indivíduos HIV soropositivos. A TB óssea, mais comum na coluna vertebral, representa $10 \%$ a $20 \%$ das lesões extrapulmonares em crianças, além de também ser mais comum em indivíduos entre 40 e 60 anos de idade (MS, 2011).

Entre todas as formas de TB, é a forma pulmonar da doença que tem especial relevância para a saúde pública. Pois, além de se tratar da forma mais frequente, é esta a que mantém a cadeia de transmissão da doença, quando bacilífera (MS, 2011).

A TBp pode ser classificada como primária, secundária ou miliar. A doença causada após o primeiro contato do indivíduo com o bacilo, primária, é mais comum em crianças, enquanto que a TBp secundária, decorrente de reativação endógena do bacilo, ou por reinfecção pela micobactéria, é mais comum em adolescentes e adultos jovens, ainda que possa ocorrer em qualquer faixa etária. A TBp miliar, forma grave da doença pulmonar, responde por $1 \%$ dos casos entre indivíduos HIV soronegativos e em 10\% dos soropositivos (MS, 2011). 
Na década de 1980 a TB chegou a ser considerada uma doença controlada, principalmente nos países desenvolvidos. Entretanto, movimentos migratórios, o envelhecimento da população, o aparecimento de bacilos multidroga resistentes e o advento síndrome da imunodeficiência adquirida (SIDA) fizeram com que a tendência epidemiológica de diminuição de casos de TB fosse alterada e a sua erradicação não tenha sido alcançada (RUFFINONETTO, 2002).

Em 1993, a Organização Mundial de Saúde (OMS) declarou a TB um problema de emergência global e, para seu controle, objetivou a implementação de novas ferramentas para diagnóstico, além de novas drogas e vacinas ante a multirresistência do bacilo, principalmente nos países onde a coinfecção com o vírus da imunodeficiência humana (HIV) era mais incidente. Com a estratégia "Stop TB" lançada pela OMS, objetivava-se provocar mudanças no panorama da TB no mundo entre os anos de 2006 e 2015. Em consonância com essa estratégia, foi lançado, pela "StopTB Partnership", em 2006 um quadro geral de ações combinadas com a estratégia "Stop TB", intitulado de "Global Plan to Stop TB", que estabeleceu como uma de suas metas de longo prazo a eliminação da TB até 2050. Essa meta estabelece uma redução da incidência de TB para menos de 1 caso em 1 milhão de habitantes (WHO, 2006).

O objetivo de parar o crescimento da taxa de incidência e invertê-la foi alcançado globalmente, principalmente em 16 dos 22 países que detinham $80 \%$ dos casos de TB no mundo. Essa taxa diminuiu aproximadamente 1,5\% ao ano desde 2000 (WHO, 2015). 
A prevalência da TB em 2015 foi reduzida à metade da observada em 1990 nas Américas, no sudeste da Ásia e no oeste da região do Pacífico, assim como em noves países com altas taxas de prevalência - Brasil, Camboja, China, Etiópia, Índia, Myanmar, Filipinas, Uganda e Vietnã. A mortalidade também foi reduzida à metade daquela observada em 1990, nas regiões das Américas, no oeste da região do Mediterrâneo, no Sudeste da Ásia e no oeste da região do Pacífico. Essa redução foi particularmente observada em onze países com elevada taxa de mortalidade - Brasil, Camboja, China, Etiópia, Índia, Mianmar, Paquistão, Filipinas, Uganda, Vietnã e Zimbábue (WHO, 2015).

Estima-se que houve 1,4 milhões de mortes por TB em 2015, e outros 0,4 milhões de mortes resultantes da coinfecção da TB e HIV. Ainda em 2015, estima-se que havia 10,4 milhões de novos casos de TB (incluindo 1,2 milhão entre pessoas HIV-positivas), dos quais 5,9 milhões correspondiam a homens, 3,5 milhões a mulheres e 1,0 milhão a crianças. Em geral, a relação masculino: feminino era de 1,6: 1. Entretanto, a infecção latente pelo bacilo da TB afeta mais de um terço da população do planeta, e uma pessoa com esta infecção pode vir a desenvolver a doença propriamente dita (WHO, 2014; WHO, 2016).

O relatório global da TB de 2016 é o primeiro produzido sob a luz dos Objetivos de Desenvolvimento Sustentável (ODSs) e da Estratégia End TB, que substituíram os Objetivos de Desenvolvimento do Milênio (2000-2015) e a Estratégia Stop TB (2006-2015), respectivamente. Os ODSs foram adotados pela ONU em setembro de 2015 e cobrem o período de 2016-2030. A estratégia End TB abrange um prazo de 20 anos (2016-2035). Ambos compartilham um objetivo comum: acabar com a epidemia global de TB. Os objetivos estabelecidos na Estratégia End TB incluem uma redução de 90\% 
nas mortes por TB e uma redução de $80 \%$ na incidência de TB até 2030 , em comparação com 2015 (WHO, 2016). Ainda cabe a observação que a substituída Estratégia Stop TB, através de sua parceria (StopTB Partnership), mantinha como uma de suas metas a eliminação da TB como problema de saúde pública até o ano de 2050, com a incidência de menos de 1 caso por 1 milhão de habitantes (WHO, 2006).

Uma nova classificação de países prioritários para a atenção e o combate à TB para o período de 2016 a 2020 também foi proposta no relatório lançado pela OMS em 2016. Essa nova classificação é composta por três listas de 30 países cada. Cada lista obedece às seguintes características epidemiológicas: carga de TB, TB multidrogarresistente e coinfecção TB/HIV. Alguns países figuram em mais de uma lista, somando assim, um total de 48 países prioritários para a abordagem da doença. O Brasil se encontra em duas dessas listas, ocupando a $20^{\underline{a}}$ posição na classificação de carga da TB e a $19^{\underline{a}}$ quanto à coinfecção TB/HIV. Vale destacar que os países que compõem essas listas respondem por $87 \%$ do número de casos de TB no mundo (WHO, 2016).

A estratégia End TB tem três indicadores de alto desempenho: a taxa de incidência, o numero absoluto de mortes por TB e a porcentagem de pacientes com TB e suas famílias que sofrem custos catastróficos como resultado da doença (WHO, 2016).

Metas para esses indicadores foram traçadas com marcos complementares de 2020 e 2025. Os marcos de 2020 e 2025 são, respectivamente, a redução de $35 \%$ e $75 \%$ do número absoluto de mortes por TB, uma redução de $20 \%$ e $50 \%$ da taxa de incidência, comparado com os 
niveis de 2015, e ainda não afetar as famílias com altos custos da doença em ambos os anos (WHO, 2016).

Para que se alcancem as metas da End TB, foram estabelecidos três pilares de atuação: 1) os cuidados e a prevenção devem estar integrados e centrados no paciente: para tanto, o diagnóstico deve ser precoce, lançando mão de testes universais de susceptibilidade a fármacos e rastreio sistemático de contatos e grupos de alto risco; além disso, o tratamento de todas as pessoas com TB deve ser amplamente disponível, incluindo TB resistente a medicamentos e deve ser oferecido o apoio necessário para que o paciente adira o tratamento; ações visando medidas colaborativas de controle e tratamento da coinfecção TB/HIV e manejo de outras comorbidades; estabelecer e promover a prevenção da TB para pessoas com maior vulnerabilidade e ampliar a vacinação contra a TB. 2) políticas públicas e sistemas de apoio de combate à doença alicerçados no compromisso político em destinar recursos adequados para cuidados e prevenção de TB; no compromisso comunitário, com participação de organizações da sociedade civil e prestadores de cuidados públicos e privados; em uma política de cobertura de saúde universalizada com notificações amplas e fidedignas de casos, melhorando a qualidade dos registros, e colaborando com o uso racional de medicamentos para o controle da infecção; e na proteção social, com a diminuição da pobreza e ações sobre outros determinantes da TB. 3) investimento em pesquisas e inovações, promovendo descobertas, desenvolvimento e rápida incorporação de novas ferramentas, intervenções e estratégias; além da otimização da implementação da estratégia End TB e avaliação de seu impacto, com intuito de promover inovações (WHO, 2014). 
Sabendo que a doença mantém estreita relação com as condições socioeconômicas da população, produto da história do desenvolvimento social e ambiental em que a mesma se insere, entende-se que pobreza, desnutrição, altas densidades demográficas, baixa escolaridade, além de condições individuais de imunossupressão estão relacionadas com o aumento dos casos da TB (HINO et al, 2011a; QUEIROGA et al, 2012).

A percepção de que os determinantes sociais influenciam a ocorrência de TB pode também ser evidenciada pela sua desigual distribuição ao redor do mundo. Notadamente, os novos casos descritos da doença continuam se concentrando nos grupos mais pobres da sociedade, com desigual acesso aos serviços de saúde (HARGREAVES et al, 2011; CDSS, 2010). Essa desigualdade é o resultado das "circunstâncias em que as populações crescem, vivem, trabalham e envelhecem" associadas aos sistemas de saúde que privilegiam o tratamento da doença em detrimento da prevenção (CDSS, 2010).

A Comissão sobre Determinantes Sociais da Saúde (CDSS) formada pela OMS define que as condições que reforçam, ou até mesmo geram, a estratificação da sociedade também determinam estruturas na saúde. Isso ocorre por desigualdade das condições materiais de vida, das diferenças de circunstâncias psicossociais, assim como da diferente exposição a riscos comportamentais e biológicos (HARGREAVES et al, 2011).

A epidemiologia da TB evidencia alguns determinantes estruturais, dentre eles, a desigualdade socioeconômica, as altas taxas de migrações, o rápido processo de urbanização e o crescimento populacional. Como consequências 
dessas condições, há uma distribuição também desigual de características sociais que influenciam a ocorrência de TB, tais como: insegurança alimentar e desnutrição, condições precárias de habitação, meio ambiente insalubre, pobreza, baixos níveis de escolaridade e dificuldade de acesso a serviços de saúde. A distribuição territorial de tais características condiz com a distribuição espacial da TB, dado que influencia a exposição e a infecção pelo patógeno, a progressão da doença, o diagnóstico e o tratamento tardios ou inadequados, a baixa adesão ao tratamento e o baixo sucesso terapêutico (CDSS, 2010).

Pressupõe-se que a moradia inadequada, com superlotação, pouca ventilação, assim como precárias condições no ambiente de trabalho, aumentam as chances de um indivíduo contrair o bacilo causador da TB. A pobreza e a desnutrição aumentam a susceptibilidade ao desenvolvimento da doença, assim como podem agravar o desfecho clínico. A dificuldade de acesso a serviços de saúde, seja pela falta de equipamentos adequados e próximos, seja pela dificuldade financeira ou de transporte para tais equipamentos, seja pela estigmatização da doença ou a falta de apoio social aos sintomáticos, podem atrasar o diagnóstico a notificação da doença e ações para o tratamento e o combate à TB (HARGREAVES et al, 2011).

Uma recente observação histórica no Brasil, demonstra que em 2011, entre todos os casos de TBp bacilífera notificados, 75,4\% dos casos evoluíram com cura e $8,9 \%$ abandonaram o tratamento. Ainda nesse ano, ocorreram 4.682 mortes por TB. Em 2012 foram registrados pelo Sistema de Informação de Agravos de Notificação (SINAN) 71.230 novos casos de TB. Destes, 40.152 casos foram classificados como pulmonar bacilíferos, aqueles que possuem cavitação pulmonar e que são capazes de produzir e aerossolizar gotículas que 
contenham o bacilo, mais comuns entre os homens nas faixas etárias entre 15 a 39 anos e 40 a 59 anos. (MS, 2014).

Em 2013, 70.372 novos casos foram relatados, colocando o Brasil na listados 22 países que concentram $80 \%$ dos casos de TB no mundo, segundo a OMS. Com a incidência de 35 novos casos por 100 mil habitantes, o país ocupa a 16ª posição dessa lista em relação ao número de casos novos. Em 2015 o Brasil apresentou 73.221 novos casos de TBp e figurava entre os 20 países que detinham 84\% dos casos de TBp (WHO, 2016), uma das doenças relacionadas à pobreza, e não se distribui igualmente em todos os territórios geográficos do país. Dos novos casos da doença, $63 \%$ estão concentrados em 181 dos 5.570 municípios brasileiros, com incidência acentuada nos grupos de maior vulnerabilidade (MS, 2015).

A população carcerária e a população com coinfecção do HIV obedecem uma outra dinâmica de infecção e desenvolvimento da doença pelo bacilo da TB em relação aos demais indivíduos, seja pela maior exposição ou pelo imunocomprometimento. Observa-se na população carcerária que as prováveis causas dessa discrepância são, além do aglomerado populacional em que habitam, causas pregressas ao encarceramento, como tabagismo, uso de entorpecentes e pobreza (MS, 2011). Em 2007, foi introduzida a informação de condição prisional na ficha de notificação de TB. Essa informação permitiu a primeira avaliação em nível nacional de casos de TBp no sistema carcerário. Dessa forma, concluiu-se que de 5\% dos casos de TBp no país correspondia essa população específica, que representa apenas $0,2 \%$ da população total (SÁNCHEZ et al, 2007; SOUZA et al, 2005). Diante dessa informação, verificou-se que a prevalência da 
doença na população carcerária no momento do ingresso ao sistema penitenciário era de $2,7 \%$, o que corrobora com a tese de que as causas pregressas ao encarceramento contribuiem para a alta prevalência de TBp na população carcerária (SÁNCHEZ et al, 2009).

A população com a co-infecção pelo HIV ou aquelas com a SIDA já instalada, também apresentam maiores taxas de incidência de TBp, quando comparada ao restante da população (COUCEIRO et al, 2011). Estima-se que a oferta de testagem para a sorologia de HIV seja em torno de $70 \%$, sendo que apenas $50 \%$ têm o resultado acessado, mantendo prevalência de $15 \%$ de positividade. A taxa de óbito ante a coinfecção HIV e TB chega a $20 \%$, sendo esta a maior causa de morte nessa população (SANCHÉZ et al, 2009).

Em função dessas características, as informações que são colhidas pelos profissionais de saúde no território geram dados que podem ser analisados com ferramentas de análise espacial, associadas aos Sistemas de Informação Geográfica (SIG). Dessa forma, pode-se produzir informações que auxiliem em tomadas de decisões na esfera da saúde pública. Esses recursos propiciam que a Vigilância Epidemiológica identifique áreas e populações mais vulneráveis ao adoecimento por TB, além de outros agravos de saúde, e ainda possam planejar ações de controle e prevenção nessas área (BARBOSA e COSTA, 2013; RABELLO, 2011).

Apesar do progresso observado ao longo dos anos, com as políticas para o controle de TB, o atual objetivo de erradicação da doença é desafiador e implica em árduo trabalho. Sobretudo porque o atraso na detecção de casos 
demonstra a ineficiência e o mal funcionamento de serviços de saúde, muitas vezes inacessíveis aos doentes. A associação da doença com o HIV, ainda que não seja o objeto desse estudo, assim como a TB multidrogarresistente, representam mais um desafio para a eliminação da doença. A atenção especial às crianças, através de ações preventivas à $T B$, a melhora dos métodos diagnósticos e disponibilidade de tratamento com drogas combinadas em dose fixa, respeitando o peso da criança e com menores efeitos colaterais que levem ao abandono do seguimento são fundamentais para eliminar a doença entre os mais novos (RAVIGLIONE et al, 2012).

A melhoria constante no combate à TB requer consistente suporte em todas as instâncias, desde a ciência básica, da pesquisa operacional à implementação das tecnologias desenvolvidas. Todas essas etapas dependem de engajamento do Governo, de organizações não governamentais e da sociedade civil para que a eliminação da TB seja alcançada ( RAVIGLIONE et al, 2012).

Com as atuais tecnologias, o objetivo de eliminar a TB até o ano de 2050, dificilmente será alcançado. Novas tecnologias devem continuar a ser desenvolvidas, a fim de monitorar e avaliar continuamente indicadores e determinantes da doença. Tais ações são essenciais para compreensão dos desafios, assim como das necessidades para atingir as metas da End TB (LÖNNROTH et al, 2010).

Dentre as tecnologias para o combate à $\mathrm{TB}$, a utilização de ferramentas de análise espacial, ainda que pouco utilizadas em serviços de gestão em saúde, podem contribuir para avaliar e identificar fatores e áreas de riscos a 
serem considerados no planejamento de políticas de saúde. Adequadamente utilizados, esses instrumentos podem melhorar os índices de detecção, diagnóstico, tratamento e cura de doenças (DOS SANTOS et al, 2014). 


\section{OBJETIVO}

\subsection{Objetivo Geral}

O presente estudo tem por finalidade descrever a distribuição espacial e temporal dos casos de TBp no ESP, entre os anos de 2007 e 2013, e identificar possíveis fatores de risco para sua ocorrência.

\subsection{Objetivos específicos}

a) descrever a ocorrência da TBp nos municípios do ESP

b) identificar aglomerados espaciais e de variação espacial das tendências temporais de municípios com alto e baixo riscos de ocorrência de TBp;

c) avaliar a relação entre a ocorrência de TBp e indicador socioeconômico e demográfico representativo da renda, longevidade e escolaridade. no espaço estudado. 


\section{MÉTODO}

\subsection{Tipo, população e área de estudo}

Trata-se de um estudo ecológico, cuja população de estudo refere-se aos casos novos de TBp residentes nos municípios do ESP e notificados ao Sistema de Informação de Agravos de Notificação (SINAN) no período de 2007 a 2013. Esse Estado localiza-se na região sudeste do Brasil (Figuras 1A e B), dividido em 645 municípios, com capital homônima e apresentou uma população de 41.262.199 habitantes no ano de 2010. A Figura 1C apresenta as mesorregiões em que estão agrupados os municípios do ESP.

\subsection{Fonte de dados}

Do Departamento de Informática do Sistema Único de Saúde (DATASUS), foram obtidos os números de casos novos de TBp, segundo as variáveis sexo, idade e município, tendo como base os casos notificados ao Sistema de Informação de Agravos de Notificação (SINAN) (DATASUS, 2016). Esse estudo não considerou algumas populações específicas, como a carcerária e indivíduos com coinfecção pelo vírus da imunodeficiência humana (HIV) e aqueles com quadro confirmado da SIDA, os quais apresentam taxa de incidência muito distinta da população geral (SÁNCHEZ et al, 2009).

Os dados foram extraídos do DATASUS, para cada ano do período estudado, obedecendo aos seguintes critérios de seleção segundo campodado: linha: município de residência; coluna: faixa etária; conteúdo: casos confirmados; faixa etária: todas as categorias; sexo: todas as categorias (em primeira seleção), feminino (em segunda seleção) e masculino (em 
terceira seleção); tipo de entrada: caso novo; institucionalizado: todas as categorias exceto presídio; forma-pulmonar: pulmonar+extra-pulmonar; Aids: ignorado/branco e não; HIV: todas as categorias exceto positivo.

Figura 1 - América do Sul, Brasil e estado de São Paulo (A); estado de São Paulo e seus municípios, com destaque para a capital paulista (B); Mesorregiões do estado de São Paulo (C).

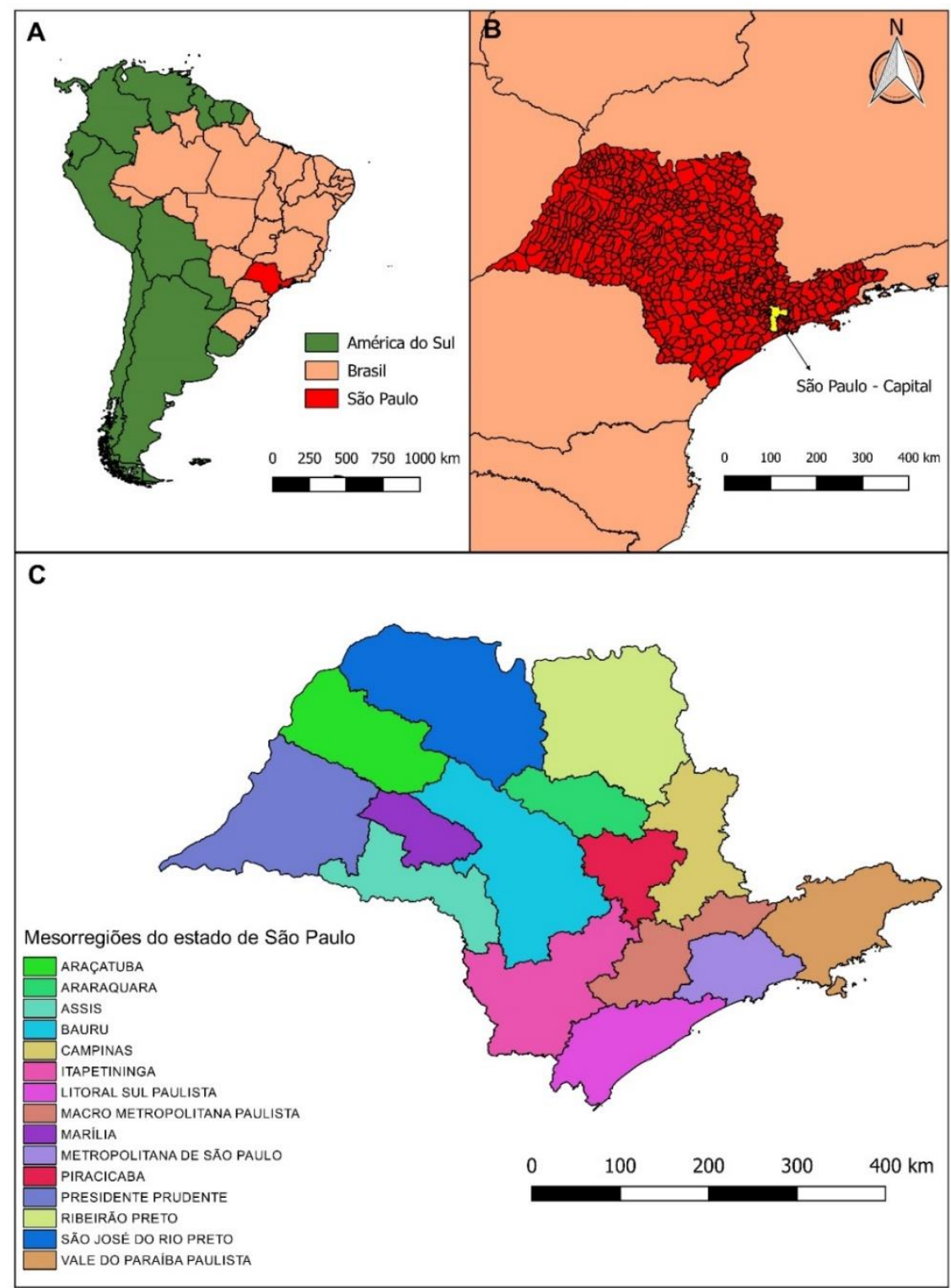


As estimativas populacionais, segundo sexo e idade, empregadas neste estudo, bem como as bases cartográficas para a produção dos mapas dos municípios, foram obtidas do Instituto Brasileiro de Geografia e Estatística (IBGE). Foi escolhido como indicador representativo das condições socioeconômicas e demográficas dos municípios paulistas o Índice Paulista de Responsabilidade Social (IPRS) referente ao ano de 2010 (meio do período), cujos valores para os municípios do ESP em 2010 (meio do período) foram obtidos junto à Fundação Sistema Estadual de Análise de Dados (SEADE)a. Este índice classifica os municípios em cinco categorias segundo as variáveis riqueza, longevidade e escolaridade. A categoria 1 representa os municípios com alta riqueza, alta ou média longevidade e alta ou média escolaridade, a categoria 5 representa municípios com baixas riqueza, longevidade e escolaridade e as demais categorias intermediários, entre estes os extremos superior e inferior do indicador.

\subsection{Georreferenciamento}

Os casos de TBp foram georreferenciados segundo município de residência tomando-se como base os códigos dos municípios presentes no banco de dados obtidos do DATASUS e os mesmos códigos disponíveis no mapa de municípios do ESP obtido do IBGE. Estes procedimentos foram realizados no programa QGIS 2.14 (QGIS DEVELOPMENT TEAM, 2016).

\subsection{Análise dos dados}

Foram calculadas taxas de incidência de TBp para todo o ESP e segundo faixas etárias e sexo para todo o período de estudo, considerando-

\footnotetext{
a Entenda o Índice Paulista de Responsabilidade Social - IPRS. Disponível em http://indicesilp.al.sp.gov.br/view/pdf/iprs/estado.pdf. Acessado em 30/6/2017.
} 
se a população de 2010 (meio do período). Foram calculadas taxas de incidência por TBp para O ESP (totais e por sexo) segundo ano, padronizadas diretamente, por idade e sexo, utilizando-se como padrão os dados populacionais correspondentes ao ano censitário de 2010. Estas tiveram sua tendência temporal avaliada por meio do método de PraisWinsten (nível de significância de 0,05), que leva em conta autocorrelação temporal de primeira ordem, através de pacote prais disponível no software R 3.3.3 (R DEVELOPMENT CORE TEAM, 2017).

Para a análise da ocorrência da TBp no espaço, isto é, nos municípios do ESP, foi necessário considerar a dependência espacial deste fenômeno, além do excessivo número de zeros, quando a doença foi considerada por ano de ocorrência. Em geral, distribuições deste tipo têm soluções apenas no âmbito da estatística Bayesiana e, mesmo assim, com excessivo e muitas vezes proibitivo consumo de tempo computacional, caso a opção seja a modelagem usando as chamadas simulações por Markov Chain Monte Carlo (MCMC). Recentemente, foi desenvolvida a abordagem INLA (Integrated Nested Laplace Approximations) (RUE et al 2009), que é uma alternativa computacionalmente eficiente aos métodos MCMC, especialmente quando da presença de estrutura espacial (o caso deste estudo) ou espaço-temporal dos dados. O INLA, utilizando uma abordagem determinística, permite que sejam obtidas as distribuições posteriores dos parâmetros de interesse (BLANGIARDO et al, 2013).

A necessidade de considerar a dependência espacial da variável dependente em questão (a ocorrência de TBp nos municípios) está relacionada com o delineamento epidemiológico utilizado neste estudo, o 
ecológico. Em tal situação e pensando na chamada primeira lei da geografia (tudo se parece, mas as coisas mais próximas se parecem mais entre si do que as mais distantes) (TOBLER, 1970), os valores da variável dependente não são independentes uns dos outros. Esta questão, que deve ser colocada em conjunto com as decorrentes da existência de dependência temporal e a hierarquia dos dados, entre outras, relaciona-se ao problema da pseudorreplicação dos dados, que, se não levado em conta, produz modelos com estimativas enviesadas (ZUUR et al, 2017).

A ocorrência de TBp no ESP foi mapeada segundo municípios para cada ano, utilizando-se modelos Bayesianos Gaussianos latentes. Isto foi feito considerando-se que a taxa média de incidência de TBp em cada município seria função de um intercepto e duas componentes aleatórias. A primeira representaria os resíduos espacialmente estruturados, modelados por meio de uma estrutura autorregressiva condicional (iCAR), e a segunda os resíduos não estruturados (BLANGIARDO et al 2013).

Para o mapeamento da TBp por ano de estudo, o número de casos observados por município foi modelado utilizando-se as especificações propostas por Besag et al (1991), o denominado modelo de Besag-YorkMollié (BYM) e uma distribuição de Poisson inflada de zeros. Casos esperados, por município e por ano, foram obtidos por padronização indireta, tendo como base as taxas por faixa etária de todo o período de estudo e para todo o ESP, calculadas para 2010, os quais foram tomados como offset na modelagem. Com isso, foram obtidas razões de incidência padronizadas (RIP) e, em um contexto Bayesiano, as probabilidades das RIP serem maiores do que um nas unidades de análise (BLANGIARDO et al 
2013). A opção pelo uso de uma distribuição inflada de zeros resultou de quantidades de municípios com zero casos entre $30,5 \%$ e $34,3 \%$, segundo ano de estudo, e dos modelos obtidos apresentarem menores valores de DIC (Deviance Information Criterion) quando comparados com modelos obtidos apenas utilizando-se distribuição de Poisson.

Para avaliação da relação entre os casos observados de TBp em todo o período de estudo o IPRS dos municípios paulistas, utilizaram-se as mesmas especificações apresentadas acima, considerando-se que a taxa média de incidência de cada município seria função de um intercepto, das covariáveis e dos efeitos aleatórios espacialmente estruturado e não estruturado (BLANGIARDO et al 2013).

As modelagens foram realizadas utilizando-se a abordagem INLA (Integrated Nested Laplace Approximation) e disponível como um pacote do Programa R (R DEVELOPMENT CORE TEAM, 2017), o R-INLA (RUE et al 2009). Todas as modelagens consideram priores minimamente informativas.

De posse dos mapas com os casos de TBp georreferenciados por município e com vistas a aplicação de estatísticas de varredura para deteç̧ão de aglomerados espaciais e espaço-temporais de alto e baixo risco e de variação espacial da tendência temporal, foram produzidos três bancos de dados. O primeiro continha informações sobre o número de casos por ano, grupo etário e sexo segundo cada um dos municípios paulistas. O segundo banco de dados continha informações sobre as populações de cada um dos 645 municípios, segundo grupo etário e sexo. 
O terceiro banco continha informações sobre as coordenadas geográficas (latitude e longitude) dos centroides de cada um dos municípios do ESP.

Após a composição destes bancos, eles foram importados para 0 programa SaTScan 9.4 para aplicação das estatísticas de varredura e detecção de aglomerados (KULLDORFF, 1997, 2015). Para isso foram consideradas as seguintes condições: modelo de Poisson, ajuste para idade e sexo, aglomerados sem sobreposição englobando no máximo $50 \%$ da população sobre risco. Foram considerados significantes aglomerados com valores de $p$ menores do que 0,05 , sendo que estes foram obtidos pelo método de Monte Carlo com 999 replicações.

Como resultados das varreduras foram obtidos os riscos relativos (RR), ajustados por idade e sexo, representando a relação entre as incidências de TBp dentro e foram dos aglomerados. Foram obtidos também as tendências temporais de crescimento (decrescimento) internas e externas dos aglomerados de variação espacial das tendências temporais (KULLDORF, 2015).

Os resultados das modelagens e das estatísticas de varredura foram exportados para o programa QGIS 2.14 (QGIS Development Team, 2016), no qual foram elaborados os mapas temáticos.

\subsection{Aspectos éticos}

O projeto desse estudo foi submetido ao Comitê de Ética em Pesquisa da Faculdade de Saúde Pública da USP, sob número de certificado 59790216.4.0000.5421, e aprovado pelo mesmo, segundo parecer 1.738.876. 


\section{RESULTADOS E DISCUSSÃO}

Aqui apresenta-se o artigo intitulado "Tuberculose pulmonar em municípios do estado de São Paulo: avaliação espacial e temporal", elaborado em formato para submissão à Revista de Saúde Pública.

Título: Tuberculose pulmonar em municípios do estado de São Paulo: avaliação espacial e temporal

Título resumido: Tuberculose pulmonar em São Paulo

Autores: Erico Filev Maia', Inês Fronteira² ${ }^{2}$ Francisco Chiaravalloti Neto'. Instituições:

1 - Faculdade de Saúde Pública da USP.

2 - Global Health and Tropical Medicine - Instituto de Higiene e Medicina Tropical - Universidade Nova de Lisboa.

\section{RESUMO}

Objetivos: Descrever a ocorrência de tuberculose pulmonar (TBp) e sua distribuição espacial e temporal nos municípios do estado de São Paulo (ESP) e avaliar a relação desta com indicador de condições socioeconômicas e demográficas. Método: Estudo ecológico, com georreferenciamento dos novos casos de TBp ocorridos entre 2007 e 2013 nos municípios de residência dos doentes. Calcularam-se incidências, no tempo e no espaço, e produziram-se mapas temáticos, identificando áreas de maior e menor risco da ocorrência da doença. A relação entre incidência de TBp e o indicador socioeconômico e demográfico foi analisada por meio de regressão espacial. As análises estatísticas utilizaram método de Prais-Winsten, estatísticas de varredura e modelagem Bayesiana Gaussiana latente. Resultados: As taxas de incidência total e por sexo apresentaram estacionariedade entre 2007 e 2013. Homens e indivíduos entre 15 e 59 anos tinham excesso de risco de TB. Os municípios das mesorregiões Metropolitana de São Paulo e Litoral Sul Paulista apresentaram, em geral, e em todos os anos de estudo, altas probabilidades de que suas razões de incidência padronizadas fossem maiores do que a unidade e parte deles constituiu-se como um aglomerado de alto risco para ocorrência de TBp. Municípios das mesorregiões de Presidente Prudente, Bauru e Marília apresentaram tendência temporal crescente das incidências em relação ao 
restante dos municípios paulistas. Municípios com renda, escolaridade e longevidade baixas apresentaram risco para ocorrência de TBp 25,0\% superior aos municípios com alta renda e altas e médias escolaridade e longevidade. Conclusão: As taxas de incidência apresentaram, entre 2007 e 2013, estacionariedade e maiores valores para homens e pessoas entre 15 e 59 anos. Identificaram-se municípios com alto risco para ocorrência de TBp e municípios, embora com riscos médios para TBp, com tendência crescente das incidências. Municípios com piores condições socioeconômicas e demográficas relevaram-se de maior risco para ocorrência de TBp.

Palavras chave: Tuberculose, Incidência, Análise Espacial, Distribuição Temporal, Brasil.

\section{Pulmonary tuberculosis in municipalities of São Paulo state: spatial and temporal analysis}

\section{Pulmonary tuberculosis in São Paulo}

\section{ABSTRACT}

Objectives: To describe the occurrence of pulmonary tuberculosis (TBp) and its spatial and temporal distribution in the municipalities of the state of São Paulo (ESP) and to evaluate its relationship with and indicator of socioeconomic and demographic conditions. Method: Ecological study, with georeferencing of new cases of TBp occurring between 2007 and 2013 to the municipalities of residence of the patients. Incidences, in time and space, were calculated and thematic maps were produced, identifying areas of greater and lesser risk of disease occurrence. The incidence of tuberculosis was related to socioeconomic and demographic indicator by spatial regression. Statistical analyzes used Prais-Winsten method, scan statistics and latent Gaussian Bayesian model. Results: Total and by sex incidence rates showed stationarity. Men and people between 15 and 59 years old presented a risk excess to TBp. The municipalities of the São Paulo Metropolitan and Litoral Sul Paulista mesoregions presented, in general and in all the years of study, a high probability that their standardized incidence ratios would be greater than unity and part of them constituted as a cluster of high risk for occurrence of TBp. Municipalities in the Presidente Prudente, Bauru and Marília mesoregions showed an increasing temporal trend of incidence in relation to the rest São Paulo municipalities. Municipalities with low income, schooling and longevity presented a risk of occurrence of TBp $25.0 \%$ higher than municipalities with high income and high and medium schooling and longevity. Conclusion: The incidence rates between 2007 and 2013 showed stationarity and higher values for men and people between 15 and 59 years. We identified municipalities with high risk for occurrence of TBp and municipalities, although with average risks for TBp, with increasing tendency of incidence. Municipalities with worse socioeconomic and demographic conditions were found to be at greater risk for the occurrence of the disease.

Keywords: Tuberculosis, Incidence, Spatial Analysis, Temporal 
Distribution, Brazil.

\section{INTRODUÇÃO}

A tuberculose (TB) continua em posição de destaque no âmbito da saúde pública devido a ser um agravo de grande magnitude, vulnerabilidade e transcendência ${ }^{1}$.

No mundo, a TB ainda é uma das maiores causas de morte, principalmente em países pobres. Estima-se que, em 2015, tenha havido cerca de 10,4 milhões de novos casos de tuberculose no mundo ${ }^{2,3}$.

A melhoria constante no combate à TB requer consistente suporte em todas as instâncias, desde a ciência básica, da pesquisa operacional à implementação das tecnologias desenvolvidas. Essas etapas dependem de engajamento do Governo, organizações não governamentais e sociedade civil para que a eliminação da TB seja alcançada ${ }^{4}$.

Em 2015, o Brasil apresentou 73.221 novos casos de TB pulmonar (TBp) e, em 2016, figurou na vigésima posição na classificação de carga de TB entre os 30 países que detinham $87 \%$ dos casos da doença ${ }^{3}$. Trata-se de doença relacionada à pobreza e que não se distribui igualmente em todos os territórios geográficos do país. Dos novos casos da doença, $63 \%$ se concentraram em 181 dos 5.570 municípios brasileiros, com incidência acentuada nos grupos de maior vulnerabilidade ${ }^{1}$.

A Comissão sobre Determinantes Sociais da Saúde formada pela Organização Mundial de Saúde define que as condições que reforçam ou geram a estratificação da sociedade também determinam estruturas na saúde ${ }^{5}$. 
Isso ocorre por desigualdade das condições materiais de vida, das diferenças de circunstâncias psicossociais, assim como das diferentes exposição a riscos comportamentais e biológicos 5 . A vigilância em saúde assume um importante papel para o monitoramento territorial dos casos de TB, pois considera-se que a captação da demanda dos problemas em saúde deve levar em conta o espaço geográfico em que a população vive ${ }^{6}$.

Ao entender que a interação entre indivíduo e território implicam em diferentes riscos de adoecimento ${ }^{7}$ busca-se 0 mapeamento de doenças, possível através dos Sistemas de Informação Geográfica (SIG), que utilizam informações apresentadas nesses territórios ${ }^{8}$, a fim de auxiliar na formatação e análises de riscos relacionados às características populacionais e do ambiente ${ }^{9}$. A aplicação destes conceitos à análise da TB pode colaborar para a uma melhor vigilância e controle da doença.

Com as atuais tecnologias, o objetivo de eliminar a TB até 2050, dificilmente será alcançado. Novas tecnologias devem continuar a ser desenvolvidas, afim de monitorar e avaliar continuamente indicadores e determinantes da doença. Tais ações são essenciais para compreensão dos desafios, assim como das necessidades para atingir as metas do End TB ${ }^{10}$.

Os objetivos deste trabalho foram descrever a ocorrência de TBp no estado de São Paulo (ESP) segundo sexo e idade; avaliar seu comportamento no tempo; descrever sua distribuição espacial segundo municípios; identificar aglomerados espaciais e de variação espacial das tendências temporais; e avaliar a relação entre sua ocorrência nos municípios e indicador socioeconômico e demográfico representativo da renda, longevidade e 
escolaridade.

\section{MÉTODO}

Trata-se de estudo ecológico misto, analítico, cuja população de estudo refere-se aos casos de TBp residentes nos municípios do ESP e notificados ao Sistema de Informação de Agravos de Notificação (SINAN) no período de 2007 a 2013. Esse Estado localiza-se na região sudeste do Brasil (Figuras 1A e B), está dividido em 645 municípios, com capital homônima e tinha, em 2010, uma população de 41.262.199 habitantes. A Figura 1C apresenta as mesorregiões em que estão agrupados os municípios do ESP.

Do Departamento de Informática do Sistema Único de Saúde (DATASUS), foram obtidos os números de casos novos de TBp, segundo as variáveis sexo, idade e município, tendo como base os casos notificados ao SINAN ${ }^{11}$. Esse estudo não considerou algumas populações específicas, como a carcerária e indivíduos com coinfecção pelo vírus da imunodeficiência humana (HIV) e aqueles com quadro confirmado da síndrome da imunodeficiência adquirida (SIDA), os quais apresentam taxa de incidência muito distinta da população geral ${ }^{12}$.

As estimativas populacionais, segundo sexo e idade, empregadas neste estudo, bem como as bases cartográficas para a produção dos mapas, foram obtidas do Instituto Brasileiro de Geografia e Estatística (IBGE). Foi escolhido como indicador representativo das condições socioeconômicas e demográficas dos municípios paulistas o Índice Paulista de Responsabilidade Social (IPRS) referente ao ano de 2010 (meio do período), cujos valores foram obtidos junto à Fundação Sistema Estadual 
de Análise de Dados (SEADE)a . Este índice classifica os municípios em cinco categorias segundo as variáveis riqueza, longevidade e escolaridade. A categoria 1 representa os municípios com alta riqueza, alta ou média longevidade e alta ou média escolaridade, a categoria 5 representa municípios com baixas riqueza, longevidade e escolaridade e as demais categorias intermediários, entre estes os extremos superior e inferior do indicador.

Os casos de TBp foram georreferenciados segundo município de residência tomando-se como base os códigos dos municípios presentes no banco de dados obtidos do DATASUS e os mesmos códigos disponíveis no mapa de municípios do ESP obtido do IBGE. Estes procedimentos foram realizados no programa QGIS $2.14^{13}$.

Foram calculadas taxas de incidência de TBppara todo o ESP e segundo faixas etárias e sexo para todo o período de estudo, considerandose a população de 2010 (meio do período). Foram calculadas taxas de incidência por TBp para o ESP (totais e por sexo) segundo ano, padronizadas diretamente, por idade e sexo, utilizando-se como padrão os dados populacionais correspondentes ao ano censitário de 2010. Estas tiveram sua tendência temporal avaliada por meio do método de PraisWinsten ${ }^{14}$ (nível de significância de 0,05), que leva em conta autocorrelação temporal de primeira ordem, através de pacote prais disponível no software R 3.3.3 ${ }^{15}$.

\footnotetext{
a Entenda o Índice Paulista de Responsabilidade Social - IPRS. Disponível em http://indicesilp.al.sp.gov.br/view/pdf/iprs/estado.pdf. Acessado em 30/6/2017.
} 
A ocorrência de TBp no ESP foi mapeada segundo municípios para cada ano, utilizando-se modelos Bayesianos Gaussianos latentes. Isto foi feito considerando-se que a taxa média de incidência de TBp em cada município seria função de um intercepto e duas componentes aleatórias. A primeira representaria os resíduos espacialmente estruturados, modelados por meio de uma estrutura autorregressiva condicional (iCAR), e segunda os resíduos não estruturados ${ }^{16}$.

Para o mapeamento da TBp por ano de estudo, o número de casos observados por município foi modelado utilizando-se as especificações propostas por Besag et al. ${ }^{17}$, o denominado modelo de Besag-York-Mollié (BYM) e uma distribuição de Poisson inflada de zeros. Os casos esperados, por município e por ano, foram obtidos por padronização indireta, tendo como base as taxas por faixa etária de todo o período de estudo e para todo o ESP, calculadas para 2010, os quais foram tomados como offset na modelagem. Com isso, foram obtidas razões de incidência padronizadas (RIP) e, em um contexto Bayesiano, as probabilidades das RIP serem maiores do que um nas unidades de análise ${ }^{16}$. A opção pelo uso de uma distribuição inflada de zeros resultou de quantidades de municípios com zero casos entre $30,5 \%$ e $34,3 \%$, segundo ano de estudo, e dos modelos obtidos apresentarem menores valores de DIC (Deviance Information Criterion) quando comparados com modelos obtidos apenas utilizando-se distribuição de Poisson.

Para avaliação da relação entre os casos observados de TBp em todo o período de estudo o IPRS dos municípios paulistas, utilizaram-se as mesmas especificações apresentadas acima, considerando-se que a taxa 
média de incidência de cada município seria função de um intercepto, das covariáveis e dos efeitos aleatórios espacialmente estruturado e não estruturado ${ }^{16}$. As modelagens foram realizadas utilizando-se a abordagem INLA (Integrated Nested Laplace Approximation) e disponível como um pacote do Programa $R^{14}$, o $R$-INLA ${ }^{18}$. Todas as modelagens consideram priores minimamente informativas.

De posse dos mapas com os casos de TBp georreferenciados por município e com vistas a aplicação de estatísticas de varredura para deteç̧ão de aglomerados espaciais e espaço-temporais de alto e baixo risco e de variação espacial da tendência temporal, foram produzidos três bancos de dados. O primeiro continha informações sobre o número de casos por ano, grupo etário e sexo segundo cada um dos municípios paulistas. O segundo banco de dados continha informações sobre as populações de cada um dos 645 municípios, segundo grupo etário e sexo. O terceiro banco continha informações sobre as coordenadas geográficas dos centroides de cada um dos municípios do ESP.

Após a composição destes bancos, eles foram importados para 0 programa SaTScan 9.4 para aplicação das estatísticas de varredura e detecção de aglomerados ${ }^{19,20}$. Para isso foram consideradas as seguintes condições: modelo discreto de Poisson, ajuste para idade e sexo, aglomerados sem sobreposição englobando no máximo $50 \%$ da população sobre risco. Foram considerados significantes aglomerados com valores de $p$ menores do que 0,05 , sendo que estes foram obtidos pelo método de Monte Carlo com 999 replicações. 
Como resultados das varreduras foram obtidos os riscos relativos (RR), ajustados por idade e sexo, representando a relação entre as incidências de TBp dentro e foram dos aglomerados. Foram obtidos também as tendências temporais de crescimento (decrescimento) internas e externas dos aglomerados de variação espacial das tendências temporais ${ }^{20}$.

Os resultados das modelagens e das estatísticas de varredura foram exportados para o programa QGIS $2.14^{13}$, no qual foram elaborados os mapas temáticos.

Esse estudo foi submetido ao Comitê de Ética em Pesquisa da Faculdade de Saúde Pública da USP, sob número de certificado 59790216.4.0000.5421, e aprovado pelo mesmo, segundo parecer 1.738 .876 .

\section{RESULTADOS}

No período analisado foram notificados 77.390 casos novos de TBp, desconsiderados os casos em presidiários e as coinfecções com HIV/SIDA. A taxa de incidência para todo o período de estudo foi de 26,8 casos/100.000 habitantes-ano, sendo 4,2 em pessoas entre 0 a 14 anos, 33,9 entre 15 e 59 anos e 27,4 em indivíduos com 60 anos ou mais. Os homens apresentaram uma taxa de incidência padronizada de 37,5 casos/100.000 habitantes-ano, enquanto as mulheres apresentaram taxa de 15,8 , correspondendo a um excesso de risco para os homens de 21,7 casos/100.000 habitantes-ano.

As taxas de incidência padronizadas para todo o ESP segundo ano, totais e segundo sexo, são apresentadas na Figura 2. As taxas de incidência 
de TBp total e por sexo, padronizadas por idade revelaram estacionariedade temporal, uma vez que as hipóteses de tendência de crescimento (decrescimento) foram rejeitadas pelo método de Prais-Winsten. Ressaltase que as hipóteses de crescimento das taxas para o sexo masculino e total foram rejeitadas com valores de p próximos ao nível de significância, respectivamente, 0,0740 e 0,0586 .

Em geral, municípios das mesorregiões Metropolitana de São Paulo e do Litoral Sul Paulista apresentaram, em todos os anos, altas probabilidade de RIP>1, com alguns municípios alternando entre altas e médias (Figura 3). Nas mesorregiões Metropolitana Paulista, Piracicaba, Vale do Paraíba Paulista e Presidente Prudente, em geral, os municípios apresentaram médias e altas probabilidades de RIP>1. As mesorregiões de São José do Rio Preto e Ribeirão Preto foram as que apresentaram os maiores números de municípios com baixas e médias probabilidades de RIP $>1$. No restante do Estado, os municípios apresentaram médias probabilidades de RIP>1, alternado com altas e baixas probabilidades.

Foi identificado, por meio de estatísticas de varredura, um aglomerado espacial de alto risco $(R R=1,9)$ localizado na região metropolitana de São Paulo e incluindo alguns municípios do Litoral Sul Paulista (Figura 4A), composto por parte dos municípios identificados como de alta probabilidade de RIP>1 (Figura 3). Foi identificado um aglomerado de baixo risco $(R R=0,5)$ englobando o restante dos municípios do $E S P$, com exceção daqueles pertencentes às mesorregiões Metropolitana de São Paulo, Litoral Sul Paulista e Vale do Paraíba. 
A Figura 4B apresenta cinco aglomerados identificados com tendência temporal de crescimento da taxa de incidência por TBp interna significativamente diferente da externa. Dentre eles, somente o aglomerado 5 (com municípios das mesorregiões Metropolitana de São Paulo, Vale do Paraíba, Macro Metropolitana Paulista e Campinas) apresentou crescimento anual interno inferior ao externo. Destacam-se os aglomerados 2 (mesorregião de Presidente Prudente) e 4 (mesorregiões de Bauru e Marília) com tendências de crescimento internas superiores às externas. $O$ aglomerado 1, apesar de composto por dois municípios, destaca-se pelo crescimento anual acima de $100 \%$. O responsável por este incremento foi Serra Azul que começou o período com um caso de TBp em 2007 e três em 2008 e finalizou com 26 casos em 2012 e 31 em 2013.

A Tabela 1 apresenta as médias posteriores das RIP de TBp e seus respectivos intervalos de credibilidade (IC) $95 \%$, segundo as categorias do IPRS, sendo identificada como importantes para a ocorrência de TBp a categoria 5. Portanto, municípios com IPRS 5 (os mais desfavorecidos) teriam um aumento do risco de ocorrência de TBp em torno de 25,0\% em relação aos municípios com IPRS 1 (os mais favorecidos).

\section{DISCUSSÃO}

A maior incidência de TBp entre pessoas de 15 a 59 anos observada nesse estudo concorda com o descrito na literatura ${ }^{21}$. Devem ser consideradas também a diferença da exposição a fatores de risco entre homens e mulheres, a maior representatividade no mercado de trabalho dos primeiros, muitas vezes com atividades laborais em ambientes fechados, com grande concentração de 
pessoas e mal ventilados, além do deslocamento demorado nas grandes cidades em transportes coletivos lotados e também com ventilação ruim. Associa-se ainda as diferenças do modo de vida que os homens apresentam em relação às mulheres, em parte da população, como etilismo e tabagismo, além da utilização de serviços de medicina preventiva mais defitária ${ }^{22,23}$. As menores incidências entre os mais jovens e mais velhos possivelmente relacionam-se à maior proteção da vacina BCG aplicada ao nascimento, e à menor exposição ao envelhecimento ${ }^{24}$.

Cabe observar que os sintomas apresentados pelos homens são os mais bem descritos na literatura, com febre, tosse e expectoração. Possivelmente esse é um fator que influencia a maior detecção da doença em homens. Em contrapartida, as mulheres relatam mais fadiga e sintomas emocionais relacionados à doença ${ }^{25}$.

Os achados nesse estudo em relação a tendência temporal das taxas de incidência dos casos de TBp corresponde ao descrito por Galesi e Fukasava ${ }^{26}$, em que foi observada uma discreta oscilação da ocorrência e da taxa de incidência de TB no ESP entre os anos de 2007 e 2013. Além disso, eles observaram maiores taxas na região metropolitana de São Paulo e no Litoral Sul Paulista e menores no interior do Estado, resultados semelhantes aos do presente estudo ${ }^{26}$.

Guimarães et al. $^{27}$ observaram queda sistemática nas taxas de incidência, prevalência e mortalidade por TB, no Brasil e nas Américas entre os anos de 1990 e 2010. Parte desse período corresponde ao presente estudo e não concorda com a tendência estacionária para as 
incidências aqui encontradas. Destaca-se que as hipóteses de tendências crescentes das incidências total e nos homens foram rejeitadas com valores de $p$ pouco acima dos $5 \%$. Este resultado pode estar relacionado com 0 pequeno número de anos analisados, sendo, talvez, um equívoco rejeitar estas hipóteses ${ }^{28}$. Todavia, o acompanhamento destas incidências por mais anos permitirá identificar o seu real comportamento.

Galesi e Fukasava ${ }^{26}$ identificaram crescimento da porcentagem de sintomáticos respiratórios investigados para a TBp entre 2007 e 2013 no ESP, fato que poderia explicar a não ocorrência de decréscimo das incidências ou mesmo de seu aumento. De todo modo, a não ocorrência de decréscimo das incidências, como previsto pela StopTB Partnership, representa uma barreira a ser vencida para atingir as metas do EndTB no ESP.

Observando que as incidências de TBp não diminuíram, como seria de se esperar, devem ser realizados estudos no sentido de perceber quais os determinantes para este cenário. A avaliação de respostas adequadas em termos de diagnóstico e tratamento, dos serviços de saúde e das condições de vida podem ajudar a esclarecer os motivos desse achado, assim como determinar os locais onde devem ser realizados maiores esforços para melhorar tais respostas e condições.

Algumas das regiões mais populosas do ESP, como a mesorregião Metropolitana de São Paulo e Litoral Sul Paulista, são também as áreas com maior probabilidade de RIP $>1$, reforçando a hipótese de que áreas mais populosas representam maior risco de contaminação e adoecimento pela TBp ${ }^{29}$. Em contrapartida, as mesorregiões de Ribeirão Preto e São José do Rio 
Preto apresentaram baixas e médias probabilidades de $R I P>1$, o que vai de encontro com a alta cobertura do tratamento diretamente observado e com a baixa iniquidade social nos municípios destas regiões ${ }^{30}$.

Este estudo também trouxe à luz que, apesar do interior do ESP ter se apresentado com uma área de menor risco para TB, existem regiões que merecem ser priorizadas nas atividades de vigilância epidemiológica. Os aglomerados compostos por municípios das mesorregiões de Presidente Prudente, Bauru e Marília possivelmente terão sua importância aumentada no quadro epidemiológico da TBp no ESP, dadas as tendências temporais crescentes internas maiores que as externas. Uma vez identificas áreas que podem tornar-se de risco no futuro, as autoridades sanitárias devem ter uma ação proativa evitando o escalonamento no número de novas infecções. Por outro lado, o aglomerado composto por municípios das mesorregiões Metropolitana de São Paulo, Vale do Paraíba, Macro Metropolitana Paulista e Campinas, ao apresentarem tendência interna de crescimento inferior à externa, possivelmente perderá parte de sua importância epidemiológica.

O município de Serra Azul chama a atenção pelo aumento do número de casos em mais de $100 \%$ ao ano, no período estudado. Algumas hipóteses para esse achado seriam a melhora da qualidade da notificação da doença a partir de 2007, ou ainda a notificação de casos de população carcerária sem essa identificação. Serra Azul tem cerca de 11 mil habitantes e conta com duas penitenciárias, inauguradas em 2002, com população carcerária em torno de 3500 detentos $^{b}$. Uma hipótese mais pessimista seria que o quadro encontrado

\footnotetext{
${ }^{b}$ Governo do Estado de São Paulo. Secretaria de Administração Penitenciária[Internet]. 2017 [cited 2017 Jun 1]. Available from: http://www.sap.sp.gov.br/
} 
em Serra Azul é consequência do aumento da força de infecção por TBp na população não encarcerada por conta da presença de agrupamento populacional (os encarcerados) reconhecidamente sujeito a altas taxas de infecção por $\mathrm{TBp}^{12}$.

O resultado, nesse estudo, de que os municípios com as piores condições socioeconômicas e demográficas apresentam os maiores riscos para TBp vai ao encontro da consideração do agravo como fortemente relacionado à pobreza, e que as pessoas mais pobres têm maior dificuldade de acesso aos serviços de saúde ${ }^{31,32}$. Entretanto, apenas os municípios com IPRS 5 apresentaram risco aumentado para o adoecimento pela TBp. Ou seja, aparentemente, somente condições muito precárias de vida representam maior risco para altas taxas de incidência de TBp, as quais poderiam ser diminuídas com melhorias mínimas das condições de longevidade, escolaridade e riqueza.

Os municípios classificados no pior nível do IPRS são aqueles apresentam maiores níveis de desigualdade tanto em relação aos demais municípios do Estado como internamente. Desigualdades de renda, de acesso a bens e serviços, sociais, e as que comprometem à trama de interrelação de indivíduos em grupos influenciam negativamente a saúde, seja pelo estresse provocado a partir das percepções em uma sociedade desigual, seja pela falta de investimentos em infraestrutura comunitária, ou ainda pelo desgaste das relações de solidariedade e confiança entre membros da comunidade ${ }^{33}$.

A principal limitação deste estudo refere-se ao uso de dados secundários sobre a ocorrência de TBp, com problemas relacionados à subnotificação, 
erros de classificação e incompletude dos dados. Outra limitação seria a utilização de um delineamento ecológico, visto como um desenho mais adequado para a formulação de hipóteses do que para testá-las ${ }^{34}$.

Se por um lado, há limitações, por outro há fortalezas a serem destacadas. O uso da estatística de varredura permitiu identificar áreas de maior e menor risco para TBp e, especialmente, identificar aglomerados cujas incidências apresentavam tendências temporais crescentes internas maiores do que externas $^{20}$. Esta pode ser uma importante ferramenta para a vigilância epidemiológica, dado que é possível observar a variação espacial das tendências temporais e identificar regiões que, mesmo não apresentando alto risco para $T B$, merecem ser monitoradas.

Destaca-se também o uso, neste estudo, de SIG e análise espacial, especialmente os modelos Bayesianos Gaussianos latentes, com os quais foi possível lidar com a autocorrelação espacial da incidência por TBp e o excesso de zeros e a técnica de Prais-Winsten para dar conta da dependência temporal dos dados. Estas questões relacionam-se ao problema da pseudorreplicação, onde os valores das variáveis dependentes não são independentes entre si, e, quando não levando em conta, produz modelos com estimativas enviesadas ${ }^{35}$. Por último, destaca-se a utilização da abordagem INLA, recentemente desenvolvida, que é, no contexto Bayesiano, uma alternativa computacionalmente eficiente aos métodos MCMC (Markov Chain Monte Carlo), especialmente quando da presença de estrutura espacial (o caso deste estudo) ou espaço-temporal dos dados ${ }^{16}$.

\section{CONCLUSÕES}


A taxa de incidência de TBp, para todo o período de estudo, foi de 26,8 casos por 100.000 habitantes-ano, com excesso de risco para homens e pessoas entre 15 e 59 anos. As taxas de incidência para o período entre 2007 e 2013 apresentaram comportamento estacionário. Os municípios das mesorregiões Metropolitana de São Paulo e Litoral Sul Paulista foram os que apresentaram as maiores probabilidades de RIP $>1$ e parte importante deles constituiu um aglomerado de alto risco para TBp.

Municípios das mesorregiões Presidente Prudente, Bauru e Marília, mesmo tendo se configurado como aglomerados com RR em torno da unidade, mostraram tendência crescente e significante das incidências em relação ao restante do ESP. Esta seria uma indicação de que estes municípios teriam futuramente sua importância aumentada no quadro epidemiológica da TBp no ESP. Municípios classificados como de baixas renda, escolaridade e longevidade apresentaram aumento de risco para ocorrência de TBp da ordem de 25,0\% em relação aos municípios com alta renda e altas e médias escolaridade e longevidade.

\section{Referências}

1. Brasil. Ministério da Saúde. Secretaria de Vigilância em Saúde. Departamento de Vigilância Epidemiológica. Manual de recomendações para o controle da tuberculose no Brasil / Ministério da Saúde, Secretaria de Vigilância em Saúde, Departamento de Vigilância Epidemiológica. 1 ed. Brasília: Ministério da Saúde; 2011.p284.

2. WHO. Global Tuberculosis report 2014. Geneva: WHO Report; 2014. (4):171. [citado 2017 jan 13] Disponível em:http://apps.who.int/iris/bitstream/10665/137094/1/9789241564809_en g.pdf. DOI: 10.1155/2014/187842. p.171.

3. WHO. Global Tuberculosis report 2016. Geneva: WHO Report; 2016. 214. [citado 2017 jan 13] Disponível em: http://apps.who.int/iris/bitstream/10665/250441/1/9789241565394eng.pdf?ua $=1$ 
4. Raviglione M, Marais B, Floyd K, Lönnroth K, Getahun H, Migliori GB, et al. Scaling up interventions to achieve global tuberculosis control:

Progress and new developments. The Lancet. 2012;379(9829):1902-13.

DOI: $10.1016 /$ S0140-6736(12)60727-2

5. Hargreaves JR, Boccia D, Evans CA, Adato M, Petticrew M, Porter JDH. The social determinants of tuberculosis: from evidence to action. $A m \mathrm{~J}$ Public Health. 2011;101(4):654-62. DOI: 10.2105/AJPH.2010.199505

6. Hino P, Bertolozzi MR, Santos CB Dos, Takahashi RF, Villa TCS. O controle da tuberculose na perspectiva da vigilância da saúde. Esc Anna Nery - Rev Enferm. 2011;15(2):417-21.

7. Vieira R da CA, Prado TN do, Siqueira MG, Dietze R, Maciel ELN. Distribuição espacial dos casos novos de tuberculose em Vitória, Estado do Espírito Santo, no período entre 2000 e 2005. Rev Soc Bras Med Trop. 2008;41(1):826.DOI:http://dx.doi.org/10.1590/S003786822008000100017

8 Aronoff S. Geographic information systems: A management perspective. Geocarto Int [Internet]. 1989 Dec;4(4):58-58. Available from: www.cambridgema.gov/GIS/gisdata.aspx.

DOI:10.1080/10106048909354237.

9. Barcellos C, Ramalho W. Situação atual do geoprocessamento e da análise de dados espaciais em saúde no Brasil. Rev Informática Pública 2002;4(2):221-30.

10. Lönnroth K, Castro KG, Chakaya JM, Chauhan LS, Floyd K, Glaziou P, et al. Tuberculosis control and elimination 2010-50: cure, care, and social development. Lancet. 2010;375(9728):1814-29. DOI: 10.1016/S01406736(10)60483-7

11. DATASUS. Ministério da Saúde/SVS - Sistema de Informação de Agravos de Notificação - Sinan Net [Internet]. 2017. Available from: http://tabnet.datasus.gov.br/cgi/tabcgi.exe?sinannet/cnv/tubercsp.def

12. Sanchez A, Larouzé B, Espinola AB, Pires J, Capone D, Gerhardt G, et al. Screening for tuberculosis on admission to highly endemic prisons? The case of Rio de Janeiro State prisons. Int J Tuberc Lung Dis. 2009;13(10):1247-52.

13. Quantum GIS Development Team. Quantum GIS Geographic Information System. [Internet]. Open Source Geospatial Foundation Project; 2016. Available from: http://qgis.osgeo.org

14. Franz Mohr (2015). prais: Prais-Winsten Estimation Procedure for AR(1) Serial Correlation. R package version 0.1.1. http://CRAN.Rproject.org/package=prais 15. R Development Core Team. R: A language and environment for statistical computing. [Internet]. Vienna, Austria: R Foundation for Statistical Computing; 2017. Available from: http://www.r-project.org 
16. Blangiardo M, Baio G, Cameletti M, Rue H. Spatial and spatio-temporal models with R- INLA. Spat SPACIO-TEMPORAL Epidemiol [Internet]. 2013;(March 2013). Available from: http://dx.doi.org/10.1016/j.sste.2012.12.001

17. Besag, J., York, J., Mollié A. Bayesian image restoration, with two applications in spatial statistics. Annals of the institute of statistical mathematics. 1991. p. 1-59.

18. Rue H, Martino S, Chopin N. Approximate Bayesian inference for latente Gaussian models by using integrated nested Laplace approximations. J R Stat Soc B 2009; 71(2):1-35.

19. Kulldorff M. A spacial scan statistic. Communications in Statistics-Theory and methods, 26(6); 1997. p. 1481-96.

DOI:http://dx.doi.org/10.1080/03610929708831995

20. Kulldorff BM. SaTScan User Guide V9.4. 2015;1-113.

21. Hino P, Cunha TN da, Villa TCS, Santos CB dos. Perfil dos casos novos de tuberculose notificados em Ribeirão Preto (SP) no período de 2000 a 2006. Cien Saude Colet. 2011;16(suppl.1):1295-301.

22. Hino P, Takahashi RF, Bertolozzi MR, Egry EY. A ocorrência da tuberculose em um administrativo do município de são paulo. Esc Anna Nery - Rev Enferm. 2013;17(1):153-9.

DOI:http://dx.doi.org/10.1590/S1414-81452013000100021

23. Queiroga RPFD, Sá LDde, Nogueira JDA. , Lima ERVD, Silva ACO, Pinheiro PGOD, Braga JU, Distribuição espacial da tuberculose ea relação com condições de vida na área urbana do município de Campina Grande-2004 a 2007. Rev Bras Epidemiol. 2012;15(1):222-32.DOI: http://dx.doi.org/10.1590/S1415-790X2012000100020

24. Santo LALA, Santos PCH, Moreira ME. Perfil clínico, epidemiológico e laboratorial dos pacientes com tuberculose em hospital universitário da região do Vale do Paraíba , Estado de São Paulo. Bepa. 2009;6(68):1421.

25. Villa Vélez L, Patricia M Montoya A. Gender Differences in the Interpretation of Experiences of Patients with Tuberculosis in Medellín, Colombia Diferencias de género en la interpretación de las vivencias de pacientes con tuberculosis. Medellín, Colombia Gender Differences in the Interpretat. Invest Educ Enferm. 2013;31(3):217-26.

26. Galesi VMN, Fukasava S. Situação epidemiológica da tuberculose no estado de São Paulo. Bol Epidemiológico Paul. 2015;12(134):13-20.

27. Guimarães RM, Lobo ADP, Siqueira EA, Borges TFF, Melo SCC. Tuberculose, HIV e pobreza: tendência temporal no Brasil, Américas e mundo. J Bras Pneumol. 2012;38(4):511-7.

28. Ferreira JC, Patino CM. O que realmente significa o valor-p ? J Bras 
Pneumol. 2015;41(5):485. DOI:10.1590/S1806-37132015000000215.

29. Jia ZW, Jia XW, Liu YX, Dye C, Chen F, Chen CS, et al. Spatial analysis of tuberculosis cases in migrants and permanent residents, Beijing, 20002006. Emerg Infect Dis. 2008;14(9):1413-20.

DOI: $\underline{10.3201 / 1409.071543}$.

30. Yamamura M, Neto MS, Freitas IM De, Rodrigues LBB, Popolin MP, Uchoa SA Da, et al. Tuberculose e iniquidade social em saúde: uma análise ecológica utilizando técnicas estatísticas multivariadas, São Paulo, Brasil. Rev Panam Salud Publica [Internet]. 2014;35(4):270-7.

31. Onifade DA, Bayer AM, Montoya R, Haro M, Alva J, Franco J, et al. Gender-related factors influencing tuberculosis control in shantytowns: a qualitative study. BMC Public Health. 2010;10:381. DOI: 10.1186/14712458-10-381.

32. Maher D. The Global Plan to Stop TB, 2006-2015. Actions for life: Towards a world free of tuberculosis. Int J Tuberc Lung Dis. 2006;10(3):240-1.

33. Fiorati RC, Arcêncio RA, Souza LB de. Social inequalities and access to health: challenges for society and the nursing field. Rev Lat Am Enfermagem. 2016;24:e2687. DOI: 10.1590/1518-8345.0945.2687

34 Pereira MG. Epidemiologia - Teoria e Prática. Rio de Janeiro: Guanabara Koogan, 2002.

35. Zuur AF, leno EM, Saveliev AA. Beginner's Guide to Spatial, Temporal and Spatial-Temporal Ecological Data Analysis with R-INLA. Volume I: Using GLM and GLMM. Newburgh: Highland Statistics Ltd, 2017. 
Tabela 1 - Médias posteriores dos efeitos fixos para as razões de incidência padronizadas de tuberculose pulmonar (RIP) estimadas usando modelo bayesiano Besag-York-Mollié (regressão de Poisson) e seus respectivos intervalos de credibilidade de 95\% (IC 95\%) ajustadas segundo as categorias do Índice Paulista de Responsabilidade Social (IPRS), municípios do estado de São Paulo, 2007 a 2013.

\begin{tabular}{|l|c|c|}
\hline Variáveis & RIP & IC 95\% \\
\hline Intercepto & 0,49 & $0,42-0,57$ \\
\hline $\begin{array}{l}\text { Índice Paulista de Responsabilidade Social } \\
\text { (IPRS) }\end{array}$ & & \\
\hline IPRS $=1$ (Baseline) & 1,00 & \\
\hline IPRS $=2$ & 1,12 & $0,91-1,37$ \\
\hline IPRS $=3$ & 0,96 & $0,79-1,16$ \\
\hline IPRS $=4$ & 1,15 & $0,95-1,38$ \\
\hline IPRS $=5^{*}$ & 1,25 & $1,01-1,55$ \\
\hline
\end{tabular}

${ }^{*}$ Categoria considerada importante e associada à ocorrência de TBp nos municípios 


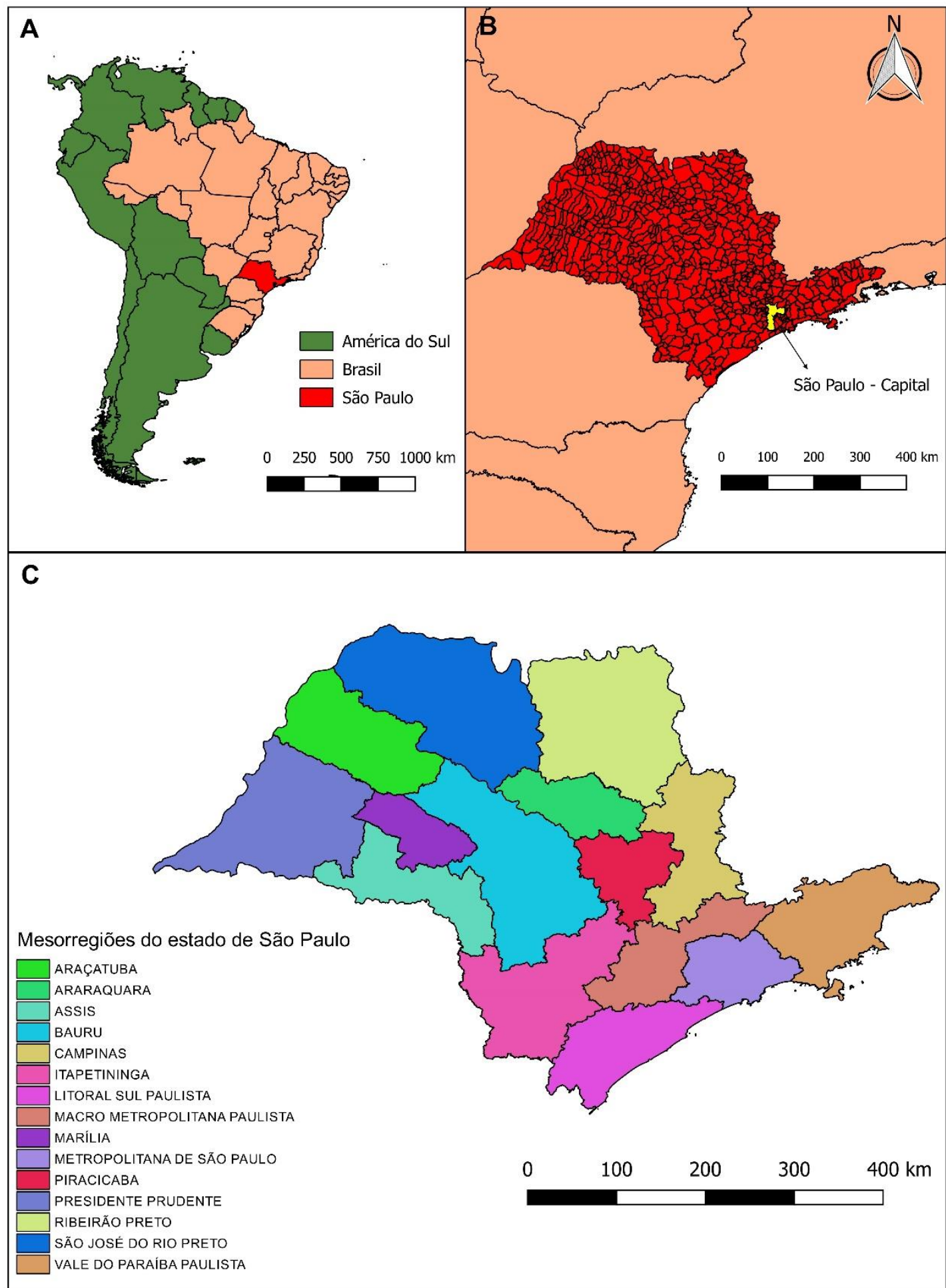

Figura 1 - América do Sul, Brasil e estado de São Paulo (A); estado de São Paulo e seus municípios, com destaque para a capital paulista (B); Mesorregiões do estado de São Paulo (C). 


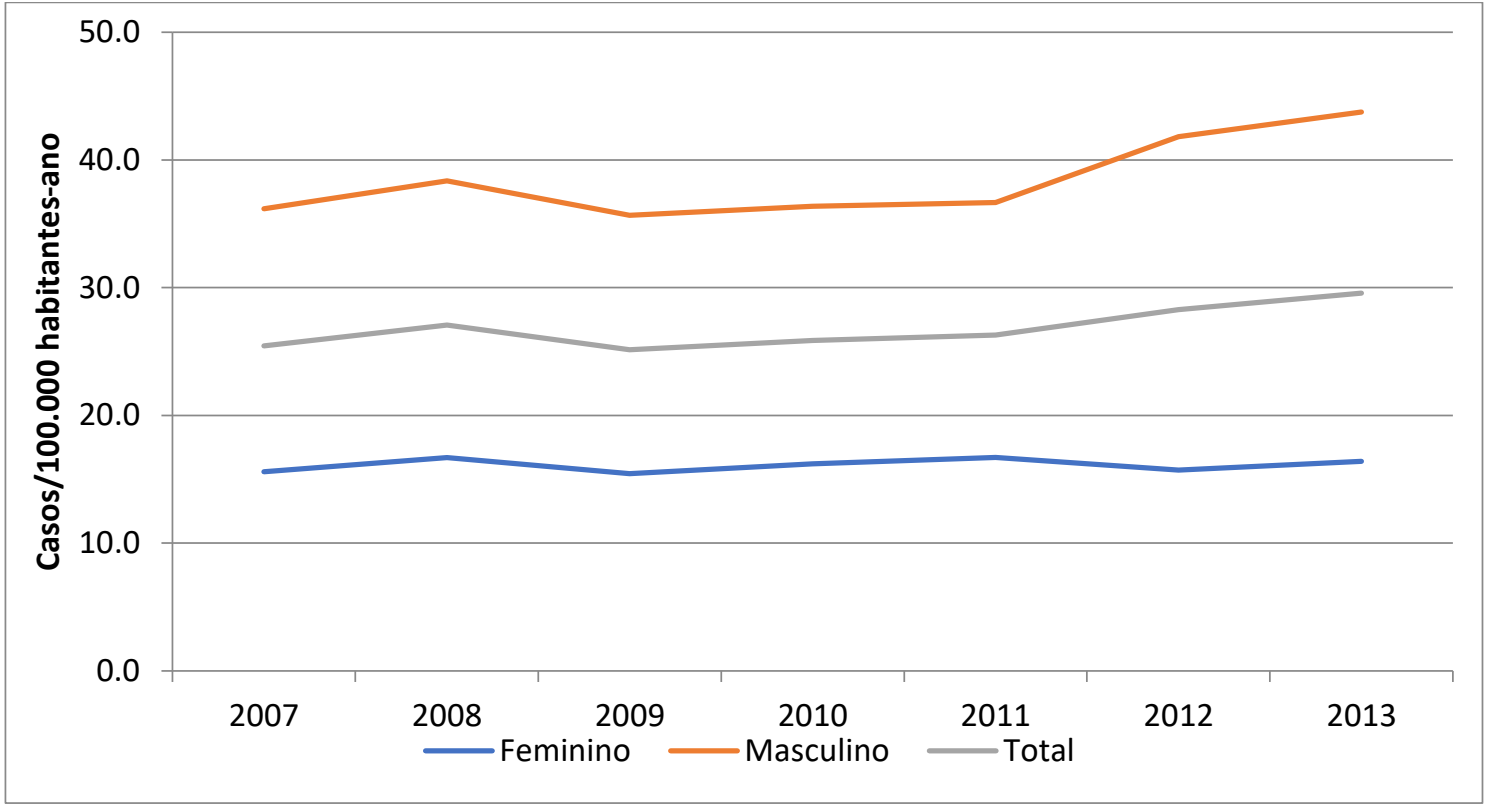

Figura 2 - Taxas de incidência de tuberculose pulmonar totais e por sexo, padronizadas por idade, segundo ano, estado de São Paulo, 2007 a 2013. 


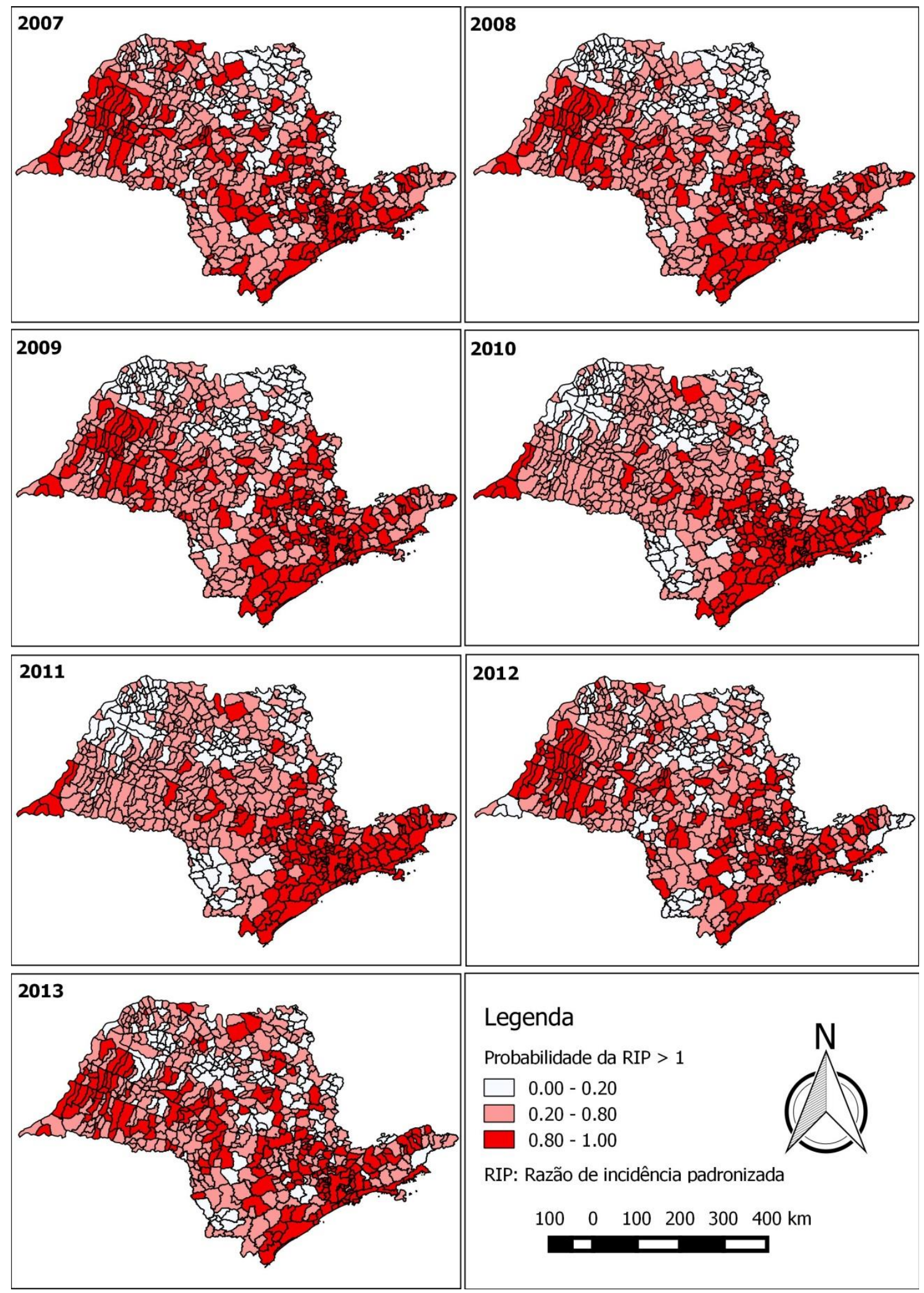

Figura 3 - Probabilidade da razão de incidência padronizada (RIP) para tuberculose pulmonar ser maior do que 1 segundo ano e município, estado de São Paulo, 2007 a 2013. 

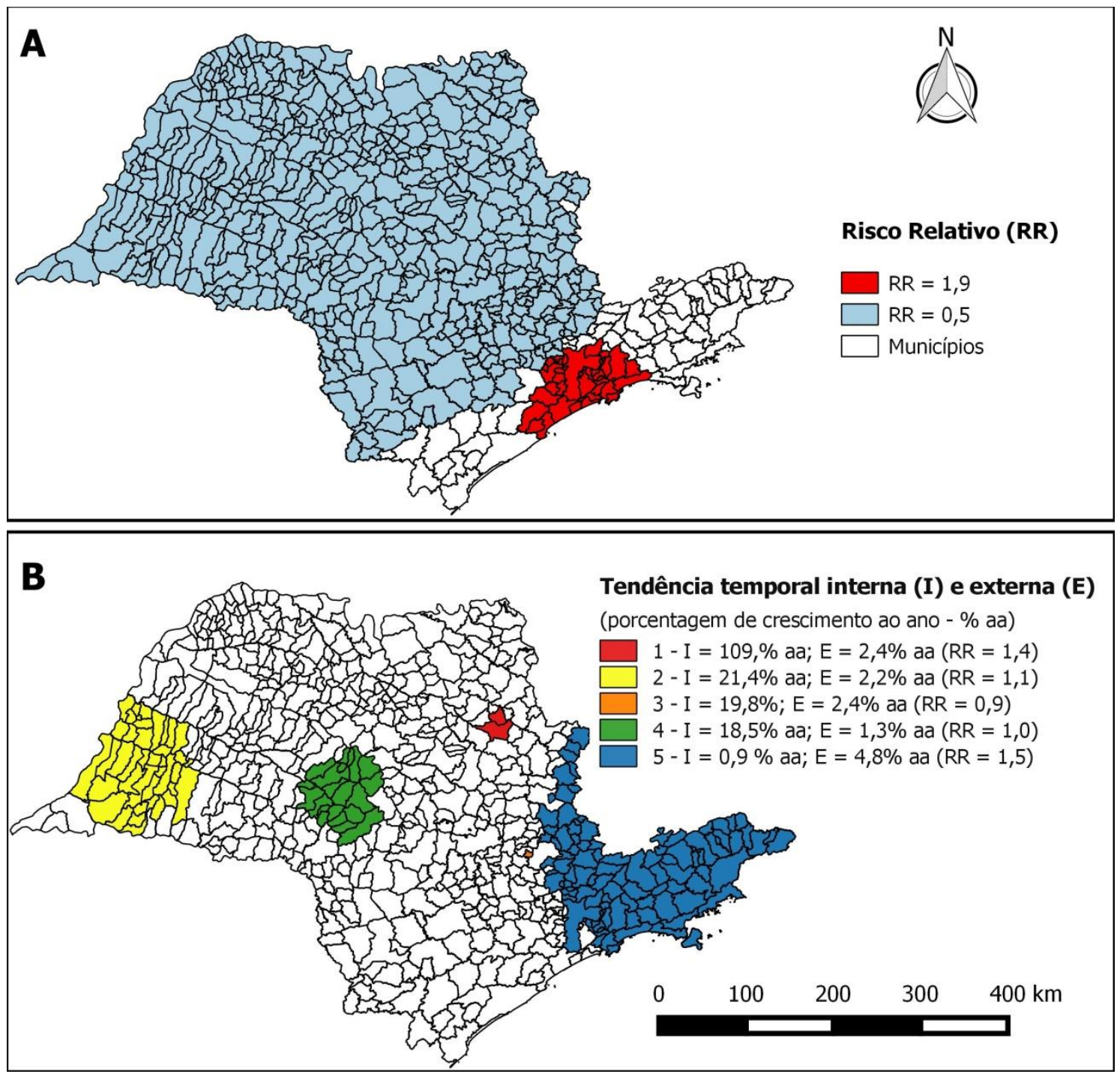

Figura 4 - (A) Aglomerados espaciais de alto e baixo risco para ocorrência tuberculose pulmonar e riscos relativos; (B) Aglomerados de variação espacial da tendência temporal para ocorrência de tuberculose pulmonar, tendências temporais internas e externas aos aglomerados e risco relativo; municípios do estado de São Paulo, 2007 a 2013. 


\section{CONCLUSÕES}

O presente estudo evidenciou aglomerados de altos e baixos riscos de ocorrência da TBp. Entre os aglomerados com mais alto risco, observam-se aqueles encontrados em parte da região Metropolitana de São Paulo e parte do Litoral Sul Paulista. Também obervamos áreas de risco relativo menor do que 1: uma área que abrange grande parte do interior do ESP.

A distribuição espacial das taxas de incidência da TBp padronizadas entre os anos de 2007 a 2013 mostra um predomínio da ocorrência da doença nas regiões metropolitana de São Paulo, na macro metropolitana paulista e no litoral sul paulista. Essas regiões foram identificadas com aglomerados de alto risco para a ocorrência da TBp.

Também foi observada a relação com significância estatística entre a ocorrência da doença e os municípios com pior distribuição de renda e IPRS mais alto.

No período estudado, há um predomínio da ocorrência da TBp na população masculina, entre 15 e 59 anos, evidenciado pelas maiores taxas de incidência nessa população e com aparente aumento dessa taxa entre os homens. Entretanto, quando analisada toda a população, as taxas de incidência mantiveram-se estáveis.

Observou-se nesse estudo que a maior parte das regiões apresentaram uma tendência temporal de queda nas taxas de incidência, exceto municípios das mesorregiões Presidente Prudente, Bauru e Marília, mesmo tendo se configurado como aglomerados com RR em torno da unidade, mostraram tendência crescente e significante das incidências em relação ao 
restante do ESP. Esta seria uma indicação de que estes municípios teriam futuramente sua importância aumentada no quadro epidemiológico da TBp no ESP. Municípios classificados como de baixas renda, escolaridade e longevidade apresentaram aumento de risco para ocorrência de TBp da ordem de $25,0 \%$ em relação aos municípios com alta renda e altas e médias escolaridade e longevidade.

A observação nesse estudo de que a tendência temporal da distribuição espacial das taxas de incidência se comporta de maneira inversa à esperada sugere que sejam feitos outros estudo para esclarecer os motivos que determinam esse cenário, assim como estudos que avaliem respostas adequadas de diagnóstico, tratamento, qualidade e oferta dos serviços de saúde, a fim de destacar regiões onde devem ser realzados maiores esforços para atingir o objetivo de eliminação da TB. 


\section{REFERÊNCIAS}

Barbosa IR, Costa ICC. Distribuição espacial dos casos novos de tuberculose no estado do Rio Grande do Norte, Brasil. Rev Baiana de Saúde Pública. 2013; 37(2):452-9.

Besag J, York J, Mollié A. Bayesian image restoration, with two applications in spatial statistics. Annals of the institute of statistical mathematics. 1991;43:159.

Blangiardo M, Baio G, Cameletti M, Rue H. Spatial and spatio-temporal models with R- INLA. Spat SPACIO-TEMPORAL Epidemiol. 2013;7:39-55.

CDSS - Comissão sobre Determinantes Sociais da Saúde. Redução das desigualdades no período de uma geração: Igualdade na saúde através da acção sobre os seus determinantes sociais: relatório final. Portugal: OMS; 2010.

Couceiro L, Santana P, Nunes C. Pulmonary tuberculosis and risk factors in Portugal: A spatial analysis. Int J Tuberc Lung Dis. 2011;15(11):1445-54.

Dos Santos NSGM, Geraldes Santos MLS, Vendramini SHF, Villa TCS, Ruffino-Netto A, Netto FC, et al. Tuberculosis and spatial analysis: Literature review [Tuberculose e análise espacial: Revisão da literatura]. Cienc y Enferm. 2014;20(2):117-29.

Ellner JJ. Tuberculose. In: L Goldman, D Ausiello (eds.) Cecil Tratado de Medicina Interna. Tradução da 24ํe ed. Rio de Janeiro: Elsevier Editora; 2014. V. 2, cap. 332.

Hargreaves JR, Boccia D, Evans CA, Adato M, Petticrew M, Porter JDH. The social determinants of tuberculosis: from evidence to action. Am J Public Health. 2011;101(4):654-62.

Hino P, Cunha TN, Villa TCS, Santos CB. Perfil dos casos novos de tuberculose notificados em Ribeirão Preto (SP) no período de 2000 a 2006. Cien Saude Colet. 2011; 16:1295-301.

Iseman MD Tuberculose. In: L Goldman, D Ausiello (eds.) Cecil Tratado de Medicina Interna. Tradução da 22º ed. Rio de Janeiro: Elsevier Editora;2005. v. 2,cap. 341 .

Kulldorff M. SaTScan User Guide v9.4. 2015;1-113.

Kulldorff M. A spacial scan statistic. Communications in Statistics-Theory and methods. 1997;26(6):1481-96.

Lönnroth K, Castro KG, Chakaya JM, Chauhan LS, Floyd K, Glaziou P, et al. Tuberculosis control and elimination 2010-50: cure, care, and social development. Lancet. 2010;375(9728):1814-29.

MS-Ministério da Saúde (BR) Manual de recomendações para o controle da tuberculose no Brasil. Brasília(DF): MS; 2011. 284 p. 
MS-Ministério da Saúde(BR). Panorama da tuberculose no Brasil Indicadores epidemiológicos e operacionais. Brasília(DF): MS; 2014.

MS-Ministério da Saúde (BR). VIGILÂNCIA EM SAÚDE Ações inovadoras e resultados. Brasília(DF): MS; 2015.

QGIS- Quantum GIS Development Team. Quantum GIS Geographic Information System. Open Source Geospatial Foundation Project; 2016.

Queiroga RPF, Sá LD, Nogueira JA, Lima ERV, Silva ACO, Pinheiro PGOD, Braga JU. Distribuição espacial da tuberculose e a relação com condições de vida na área urbana do município de Campina Grande-2004 a 2007. Rev Bras Epidemiol: 2012; 15(1):222-32.

R Development Core Team. R: A language and environment for statistical computing. Vienna, Austria: R Foundation for Statistical Computing; 2017.

Rabello RS. Tuberculose no Brasil: distribuição espacial e temporal de casos novos notificados pelo SINAN no período de 2001 a 2007. 2011.Dissertação: [Mestrado na Área de Epidemiologia em Saúde Pública], Fundação Oswaldo Cruz, Rio de Janeiro.

Raviglione M, Marais B, Floyd K, Lönnroth K, Getahun H, Migliori GB, et al. Scaling up interventions to achieve global tuberculosis control: Progress and new developments. The Lancet. 2012;379(9829):1902-13.

Rue H, Martino S, Chopin N. Approximate Bayesian inference for latente Gaussian models by using integrated nested Laplace approximations. J R Stat Soc B 2009; 71(2):1-35.

Ruffino-Neto A. Tuberculose: a calamidade negligenciada. Rev. Soc. Bras. Med. Trop. 2002; 35: 51-8.

Sánchez AR, Larouzé B, Espinola AB, Pires J, Capone D, Gerhardt G, et al. Screening for tuberculosis on admission to highly endemic prisons? The case of Rio de Janeiro State prisons. Int J Tuberc Lung Dis. 2009;13(10):1247-52.

Sánchez AR, Massari V, Gerhardt G, Barreto AW, Cesconi V, Pires J et al. A tuberculose nas prisões do Rio de Janeiro, Brasil : uma urgência de saúde pública. Cad Saúde Publica. 2007;23(3):545-52.

Souza WV, Albuquerque MFM, Barcellos CC, Ximenes RAA, Carvalho MS. Tuberculose no Brasil: Construção de um sistema de vigilância de base territorial. Rev Saude Publica. 2005;39(1):82-9.

Tobler WR. A computer movie simulating urban growth in the Detroit region. Economic Geography. 1970; Supplem: 234-40.

WHO - World Health Organization. Global tuberculosis report 2014. Geneva: WHO; 2014.

WHO - World Health Organization. Global tuberculosis report 2015. Geneva: WHO; 2015. 
WHO - World Health Organization. Global tuberculosis report 2016. Geneva: WHO; 2016.

WHO - World Health Organization. Stop TB Partnership. The global plan to stop TB, 2006-2015. Actions for life: towards a world free of tuberculosis. Geneva: WHO; 2006.

Zuur AF, leno EM, Saveliev AA. Beginner's Guide to Spatial, Temporal and Spatial-Temporal Ecological Data Analysis with R-INLA. Volume I: Using GLM and GLMM. Newburgh: Highland Statistics Ltd, 2017. 


\section{ANEXOS}

ANEXO A - Aprovação do Comitê de Ética em Pesquisa

\section{FACULDADE DE SAÚDE PÚBLICA DA UNIVERSIDADE DE SÄO PAULO}

\section{PARECER CONSUBSTANCIADO DO CEP}

DADOS DO PROJETO DE PESQUISA

Título da Pesquisa: Distribuiçäo espacial dos casos de tuberculose pulmonar nos municípios do Estado de Säo Paulo, Brasil, nos anos de 2007 a 2013.

Pesquisador: Erico Filev Maia

Área Temática:

Versäo: 1

CAAE: 59790216.4 .0000 .5421

Instituição Proponente: Faculdade de Saúde Pública da Universidade de São Paulo - FSP/USP

Patrocinador Principal: Financiamento Próprio

DADOS DO PARECER

Número do Parecer: 1.738.876

Apresentação do Projeto:

Trata-se de um estudo ecológico sobre tuberculose pulmonar nos municípios do Estado de Säo Paulo. Serão utilizados dados do Sistema de Informação de Agravos de Notificação. Os casos de tuberculose pulmonar seräo geocodificados segundo município de residència e serão calculadas as taxas de incidència de 2007-2013. Seräo construídos mapas temáticos, identificando áreas de maior e menor risco da ocorrência da doença. A detecção de aglomerados será feita por meio de estatísticas de varredura. Utilizando-se técnicas de regressäo espacial, as incidências de tuberculose seräo relacionadas a variáveis ambientais e socioeconômicas.

Objetivo da Pesquisa:

O presente estudo, tomando como unidade os municípios paulistas, tem por objetivo descrever a distribuição espacial e espaço-temporal dos casos de tuberculose pulmonar no estado de São Paulo, entre os anos de 2007 e 2013, e identificar possíveis fatores de risco para sua ocorrência.

Avaliação dos Riscos e Benefícios:

Os riscos são considerados mínimos, uma vez que serão mantidos em confidencialidade os dados individuais daqueles que desenvolveram a doença pulmonar e cujos casos foram notificados no período estudado.

Benefícios: contribuição para aprimoramento de políticas públicas de saúde com vistas à

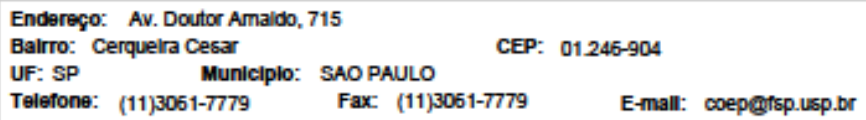




\section{FACULDADE DE SAÚDE PÚBLICA DA UNIVERSIDADE DE SÃO PAULO}

Contlnuaçlo do Parecer. 1.738.876

prevençäo e controle da tuberculose pulmonar.

Comentários e Consideraçōes sobre a Pesquisa:

Pesquisa relevante com metodologia adequada.

Consideraçöes sobre os Termos de apresentação obrigatória:

Todos os dados utilizados serão secundários, obtidos de bancos do SINAN, do DATASUS e do IBGE. Näo haverá em nenhum caso o contato pessoal com os indivíduos que tenham desenvolvido TB pulmonar no período estudado. Os casos serão geocodificados segundo município de residência de forma que não será possivel a identificação do individuo.

Conclusőes ou Pendências e Lista de Inadequaçöes:

Aprovado.

Consideraçöes Finais a critério do CEP:

Projeto aprovado.

Este parecer foi elaborado baseado nos documentos abaixo relacionados:

\begin{tabular}{|l|l|c|l|c|}
\hline \multicolumn{1}{|c|}{ Tipo Documento } & \multicolumn{1}{|c|}{ Arquivo } & Postagem & Autor & Situaçäa \\
\hline $\begin{array}{l}\text { Informações Básicas } \\
\text { do Projeto }\end{array}$ & PB_INFORMAÇŌES_BÁSICAS_DO_P & $\begin{array}{c}04 / 09 / 2016 \\
20: 25: 25\end{array}$ & & Aceito \\
\hline Rrojeto Detalhado I & Projeto_.pdf & $\begin{array}{c}31 / 03 / 2016 \\
20: 01: 58\end{array}$ & Erico Filev Maia & Aceito \\
Brochura & & & & \\
Investigador & & $\begin{array}{c}31 / 03 / 2016 \\
19: 38: 35\end{array}$ & Erico Filev Maia & Aceito \\
\hline Folha de Rosto & Follha_de_rosto.pdf & & \\
\hline
\end{tabular}

Situação do Parecer:

Aprovado

Necessita Apreciaçäo da CONEP:

Näo

SAO PAULO, 21 de Setembro de 2016

Assinado por:

Flavia Mori Sarti

(Coordenador)

Enderego: Av. Doutor Amaldo, 715

Bairro: Cerquelra Cesar

Municipio: SAO PAULO

UF: SP

Telefone: (11)3061-7779 Fax (11)3061-7779

E-mall: coep@tsp.usp.br 
ANEXO B - Confirmação da submissão do artigo à Revista de Saúde Pública

\section{Revista de Saúde Pública - Manuscript ID RSP-2017-0374}

1 message

Revista de Saúde Pública <onbehalfof+rspline+fsp.usp.br@manuscriptcentral.com>

Reply-To: rspline@fsp.usp.br

Fri, Jul 21, 2017 at 3:24 PM

To: franciscochiara@usp.br

Cc: erico.maia@usp.br, ifronteira@ihmt.unl.pt, franciscochiara@usp.br

21-Jul-2017

Dear Dr. Chiaravalloti-Neto:

Your manuscript entitled "Tuberculose pulmonar em municípios do estado de São Paulo: avaliaçäo espacial e temporal" has been successfully submitted online and is presently being given full consideration for publication in the Revista de Saúde Pública.

Your manuscript ID is RSP-2017-0374.

Please mention the above manuscript ID in all future correspondence or when calling the office for questions. If there are any changes in your street address or e-mail address, please log in to ScholarOne Manuscripts at https://mc04. manuscriptcentral.com/rsp-scielo and edit your user information as appropriate.

You can also view the status of your manuscript at any time by checking your Author Center after logging in to https://mc04.manuscriptcentral.com/rsp-scielo.

Thank you for submitting your manuscript to the Revista de Saúde Pública.

Sincerely.

Revista de Saúde Pública Editorial Office 


\section{Anexo C - Currículo Lattes de aluno e orientador}

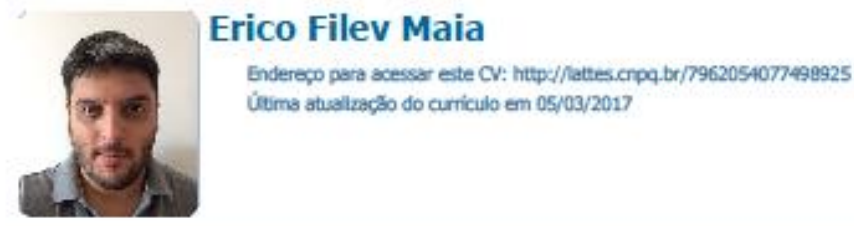

Possui graduação em Medicina pela Faculdade de Medicina da Universidade de São Paulo (2010), CRM 148.086, pós graduação em Saúde da Famlia pela Universidade Federal de São Paulo.Tem experiência na área de Medicina de Familia e Comunidade, atenção domiciliar. Atualmente trabalha com a empresa CSE Cinica Médica e na Universidade da Cidade de São Paulo como Professor/Preceptor dos alunos do sexto ano do curso de Medicina. (Texto informado pelo autor)

Identificação
$\begin{aligned} & \text { Nome } \\ & \text { Nome em citaçōes bibliográficas }\end{aligned}$
MUIA, E. F.

\section{Endereço}

Formação acadêmica/titulação

\begin{tabular}{|c|c|}
\hline 2015 & $\begin{array}{l}\text { Mestrado em andamento em Saúde Pública (Conceito CAPES 6). } \\
\text { Universidade de Săo Paulo, USP, Brasil. } \\
\text { Titulo: Análise Espacial de Casos de Tuberculose no Estado de Säo Paulo entre os anos de } \\
2007 \text { a 2013,0rientador: } 19 \text { ) Francisco Chiaravalloti Neto. }\end{array}$ \\
\hline $2012-2013$ & $\begin{array}{l}\text { Espedalizaçăo em Saúde da Familia. (Carga Horárla: } 448 \mathrm{~h} \text { ). } \\
\text { Universidade Federal de Săo Paulo, UNIFESP, Brasil. } \\
\text { Título: Neoplasias pulmonar, colonetal e gástrica: rastreamento na Atençoo Primária à } \\
\text { Saúde.. } \\
\text { Orientador: Ana Cláudia Tolentino Pires. }\end{array}$ \\
\hline $2004-2010$ & $\begin{array}{l}\text { Graduaç̆o em Medicina. } \\
\text { Faculdade de Medicina da Universidade de Säo Paulo, FMusp, Brasa. }\end{array}$ \\
\hline
\end{tabular}

Formação Complementar

\begin{tabular}{|c|c|}
\hline $2014-2014$ & $\begin{array}{l}\text { SAVC - AHA. (Carga horária: 16h). } \\
\text { Universidade Federal de Săo Paub, UNIFESP, Brasil. }\end{array}$ \\
\hline $2012-2013$ & $\begin{array}{l}\text { Especializaçăo em Saúde da Familia. (Carga horária: 48sh). } \\
\text { Universidade Federal de Săo Paulb, UNIFESP, Brasil. }\end{array}$ \\
\hline $2012-2012$ & $\begin{array}{l}\text { Promoça do Aleitamento Matemo e Alimentaçăo Comp. (Carga horária: 5h) } \\
\text { Mnistério da Saúde, MS, Brasl. }\end{array}$ \\
\hline $2008-2009$ & $\begin{array}{l}\text { Liga Academica de Urologa. } \\
\text { Faculdade de Medicina da Universidade de Sto Paulo, FMUSP, Brasil. }\end{array}$ \\
\hline
\end{tabular}

\section{Atuação Profissional}

Universidade Cidade de São Paulo, UNICID, Brasil.

Vinculo institucional 2017 - Atual 


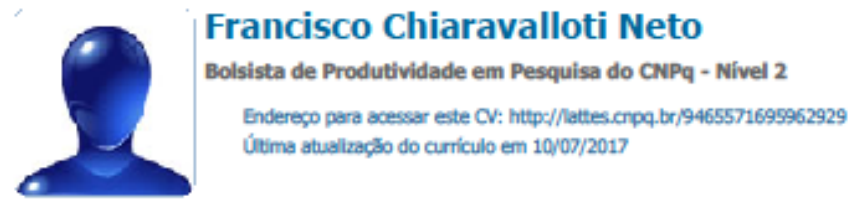

Possui graduação em Engenharia pela Escola Politécnica da USP (1981), especialização (1985), mestrado (1993) e doutorado (1999) em Saúde Pública pela Faculdade de Saúde Pública da USP (FSP-USP). Obteve, em 2010, o título de Livre Docência pela FSP-USP. É Professor Associado do Departamento de Epidemiologia da FSPUSP. Desenvolve, projetos de pesquisa na área de Epidemiologia de Doenças Transmissiveis e, em especial, as de Agentes Transmitidos por Vetores e na área de Análise Espacial em Saúde. É cadastrado com orientador nos Programas de Pós-Graduação em Saúde Pública, Programa de Pós-Graduação em Epidemiologia e no Programa de Pós-Graduação em Entomologia em Saúde Pública (Mestrado Profissional), todos na PSP-USP. (Texto informado pelo autor)

\section{Identificação}

Nome em citaçő́es bibliográficas

Francisco Chiaravallotis Neto

GIIARAVALLOTI NETO, F.; Chiaravalloti-Neto, Francisco; Chiaravalloti Neto,

Frandisco;NETO, FRANCISCO;NETO, FRANCISCO CHIARAVALLOTI; Frandisco Chiaravalloti Neto; Neto FC; francisco Chiaravalotti Neto;OHIARAVALOTTI NETO, FRANCISCO

\section{Endereço}

Endereço Profissional Universidade de Ş̆o Paulo, Faculdade de Saúde Púbäca, Departamento de Epidemiologia. Av. Dr. Arnaldo, 715

01246-904 - Sao Paulo, SP - Brasit

Telefone: (11) 30617920

Formação acadêmica/titulação

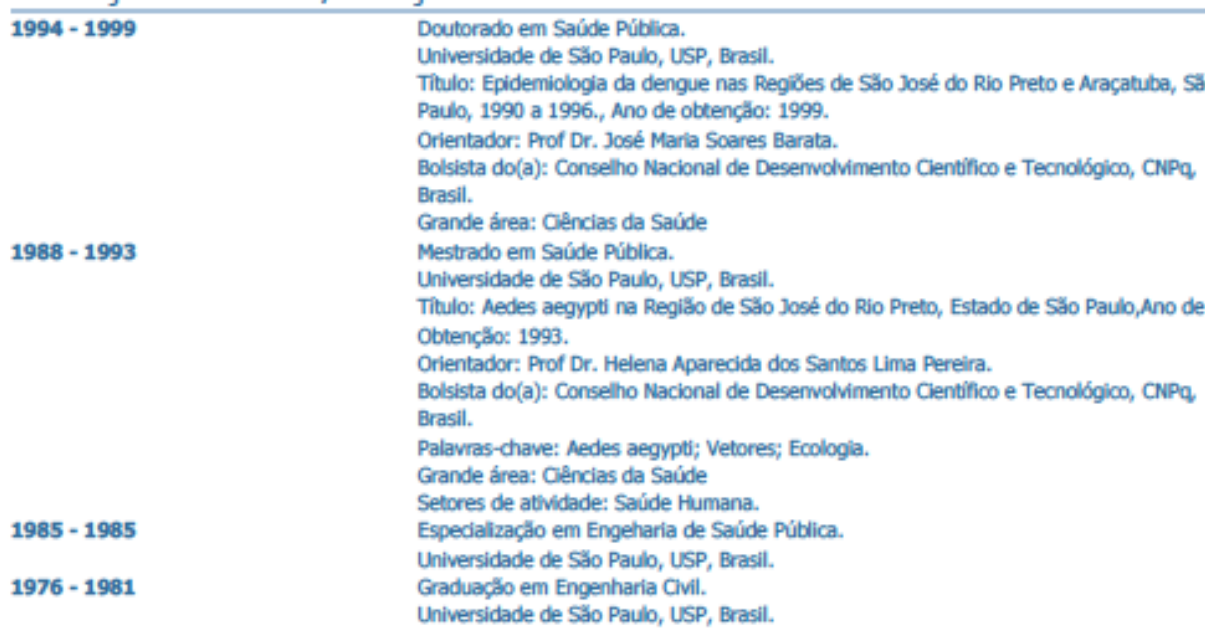

Livre-docência

\begin{tabular}{|c|c|}
\hline 2010 & $\begin{array}{l}\text { Uivre-docência. } \\
\text { Faculdade de Saúde Pública, FSP, Brasil. } \\
\text { Titulo: Estudo da cocorrência de dengue em cidade de porte médio do estado de Säo Paulo, } \\
\text { Ano de obtençăo: } 2010 . \\
\text { Palavras-chave: dengue; Epidemiologia; Análise Espacial. } \\
\text { Grande área: Cências da Saúde }\end{array}$ \\
\hline
\end{tabular}

